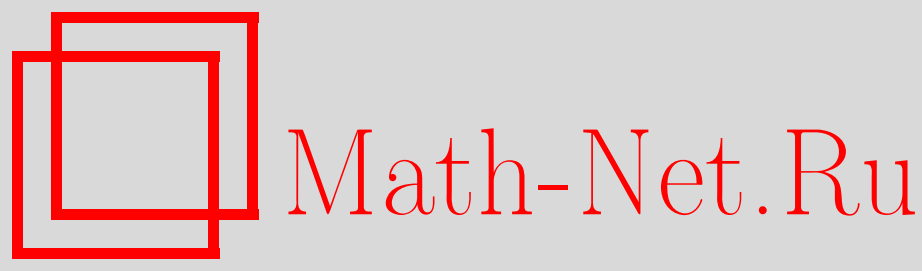

А. В. Домрин, Пространства модулей решений некоммутативной сигма-модели, ТM $\Phi, 2008$, том 156 , номep 3, 307-327

DOI: https://doi.org/10.4213/tmf6250

Использование Общероссийского математического портала Math-Net.Ru подразумевает, что вы прочитали и согласны с пользовательским соглашением http://www.mathnet.ru/rus/agreement

Параметры загрузки:

IP: 54.147 .182 .235

26 апреля 2023 г., 16:24:55

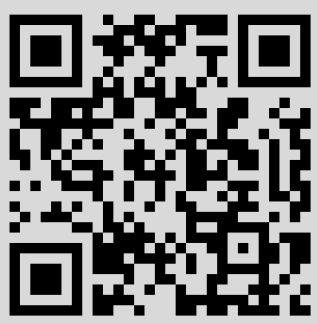




\title{
ПРОСТРАНСТВА МОДУЛЕЙ РЕШЕНИЙ НЕКОММУТАТИВНОЙ СИГМА-МОДЕЛИ
}

\begin{abstract}
Некоммутативная версия теории унитонов применяется для изучения пространства решений некоммутативной $U(1)$ сигма-модели, представимых в виде конечномерного возмущения тождественного оператора. Основными целочисленными характеристиками таких решений являются нормированная энергия $e$, канонический ранг $r$ и минимальное унитонное число $u$, всегда удовлетворяющие неравенствам $r \leqslant e$ и $u \leqslant e$. Описание начинается с так называемых BPS-решений $(u=1)$, а затем полностью описываются множества всех решений с $r=1,2, e-1, e$ (тогда автоматически $u \leqslant 2$ ) и всех решений малой энергии $(e \leqslant 5)$. Полученные результаты вскрывают простую, но не тривиальную структуру пространств модулей и приводят к серии гипотез.
\end{abstract}

Ключевые слова: некоммутативная сигма-модель, теория унитонов.

\section{1. ВВЕДЕНИЕ}

Двумерные нелинейные сигма-модели - это теории поля, конфигурационное пространство которых состоит из гладких отображений $\varphi: \mathbb{R}^{2} \rightarrow N$ в фиксированное риманово многообразие $N$ неотрицательной кривизны, а плотность лагранжиана равна квадрату модуля дифференциала отображения $\varphi$. Поэтому решения конечной энергии представляют собой гармонические отображения из $\mathbb{R}^{2}$ (или, эквивалентно, из $\left.S^{2}=\mathbb{C P}^{1}=\mathbb{R}^{2} \cup\{\infty\}\right)$ в $N$. Физические и математические аспекты таких теорий обсуждаются, например, в [1], [2]. Пространства модулей решений наиболее интересных сигма-моделей являются простыми, но не тривиальными. Это имеет место для $N=\mathbb{C} P^{1}$ (или, эквивалентно, $\left.N=U(2)\right)$ и в меньшей степени для $N=\mathbb{C} P^{n}$ с $n \geqslant 2, N=U(3)$ и $N=U(4)$.

Некоммутативная версия нелинейной сигма-модели с $N=U(n)$ появилась в [3] в связи с теорией струн и далее изучалась в [4], [5]. Информация о пространствах модулей ее решений важна для многих целей, включая квантование некоммутативных теорий поля. Некоторые способы построения решений были успешно перенесены с коммутативного случая на случай некоммутативной $U(n)$ сигма-модели [3], [6], но

${ }^{*}$ Московский государственный университет им. М. В. Ломоносова, Москва, Россия. E-mail: domrin@mi.ras.ru 
они заведомо не охватывают всего многообразия некоммутативных решений. В настоящей работе рассматривается некоммутативная $U(1)$-модель, важность которой уже отмечалась в [4]. Теория унитонов из [5] позволяет описать пространства модулей решений малой энергии: множество решений нормированной энергии $e \leqslant 5$ состоит из $\mathbb{C}^{e}$ и еще $[(e-1) / 2]$ неприводимых компонент комплексных размерностей $e-1, e-2, \ldots$. Весьма правдоподобно, что это верно и для всех $e$. Чтобы дать более подробное описание полученных результатов (которое подытожено ниже в разделе 8), напомним некоторые обозначения и результаты работы [5].

1.1. Обозначения и предварительные сведения. Рассмотрим сепарабельное гильбертово пространство $H$ с ортонормированным базисом $\left\{e_{0}, e_{1}, \ldots\right\}$. Стандартный оператор уничтожения $a e_{j}:=\sqrt{j} e_{j-1}, j=0,1,2, \ldots$, можно рассматривать как линейное отображение векторного пространства $D_{\infty}:=\left\{x \in H \mid \sum_{j=0}^{\infty}(j+1)^{k} \times\right.$ $\left|x_{j}\right|^{2}<\infty$ для всех $\left.k=0,1,2, \ldots\right\}$ в себя. То же самое верно для оператора рождения $a^{*} e_{j}:=\sqrt{j+1} e_{j+1}, j=0,1,2, \ldots$. Некоммутативная $U(1)$ сигма-модель, изучавшаяся в [4], [5], задается функционалом энергии

$$
4 e(\Phi):=\left\|\partial_{+} \Phi\right\|_{\mathrm{HS}}^{2}=\left\|\partial_{-} \Phi\right\|_{\mathrm{HS}}^{2}=\left\|\Phi_{+}\right\|_{\mathrm{HS}}^{2}=\left\|\Phi_{-}\right\|_{\mathrm{HS}}^{2}
$$

на множестве $\mathcal{M}$ всех унитарных операторов $\Phi: H \rightarrow H$ вида $\Phi=e^{i \theta} I+K$, где $\theta \in \mathbb{R}, I$ - тождественный оператор, а $K: H \rightarrow H$ - такой линейный оператор, что образ $\operatorname{im} K:=K(H)$ является конечномерным подпространством в $D_{\infty}$. Здесь $\partial_{+} \Phi:=-\left[a^{*}, \Phi\right]$ и $\partial_{-} \Phi:=[a, \Phi]$ суть некоммутативные аналоги производных по $z$ и $\bar{z}$, через $\Phi_{ \pm}:=\Phi^{-1} \partial_{ \pm} \Phi$ обозначены аналоги соответствующих логарифмических производных, а $\|X\|_{\mathrm{HS}}^{2}:=\operatorname{Tr}\left(X^{*} X\right)$ означает квадрат нормы Гильберта-Шмидта любого оператора $X: H \rightarrow H$ с конечномерным образом. Уравнения Эйлера-Лагранжа функционала (1.1) можно записать в виде $\partial_{-} \Phi_{+}+\partial_{+} \Phi_{-}=0$ или $\left[\Phi_{+}, \Phi_{-}+2 a\right]=0$ или многими другими эквивалентными способами (см. [5], утверждения 2, 3 и формула $(3.10))$. Число $e(\Phi)$ является неотрицательным и целым для любого решения $\Phi \in \mathcal{M}$ (см. [5], утверждение 12). Поэтому множество всех решений распадается на счетное число компонент, занумерованных значением $e$. Ниже будет показано, что множество решений энергии $е$ линейно связно при $e \leqslant 5$. Очень правдоподобно, что это верно и для всех $e$.

Решение $\Phi \in \mathcal{M}$ называется грассмановым, если $\Phi=\Phi^{*}$ или, эквивалентно, $\Phi=I-2 P$ для некоторого ортопроектора $P: H \rightarrow H$. Часто удобно отождествлять оператор $\Phi=I-2 P$ с замкнутым векторным подпространством $V=\operatorname{im} P$. Заметим, что ортопроектор $P: H \rightarrow H$ удовлетворяет условию $I-2 P \in \mathcal{M}$ тогда и только тогда, когда либо $\operatorname{im} P$, либо $\operatorname{ker} P$ является конечномерным подпространством в $D_{\infty}$. Топологический заряд такого проектора определяется формулой

$$
Q(P):=\left\{\begin{array}{lll}
\operatorname{dimim} P, & \text { если } & \operatorname{dimim} P<\infty, \\
-\operatorname{dim} \operatorname{ker} P, & \text { если } & \operatorname{dim} \operatorname{ker} P<\infty .
\end{array}\right.
$$

Топологический заряд грассманова решения $\Phi=I-2 P$ определяется как $q(\Phi):=$ $Q(P)$. Целое число $\delta(\Phi):=e(\Phi)-|q(\Phi)|$ является неотрицательным и четным для 
любого грассманова решения $\Phi \in \mathcal{M}$ (см. [5], утверждение 15). Это число называется дефектом решения $\Phi$. Если одно из пространств im $P$, $\operatorname{ker} P$ содержится в $D_{\infty}$ и инвариантно относительно $a$, то $\Phi=I-2 P$ является решением с $\delta(\Phi)=0$. Обратно, любое грассманово решение дефекта 0 может быть получено таким образом. Соответствующие решения $\Phi=I-2 P$ называются $B P S$-решениями (если $a(\operatorname{im} P) \subset \operatorname{im} P)$ или анти-BPS-решениями (если $a(\operatorname{ker} P) \subset \operatorname{ker} P)$. В нашем контексте нет необходимости отдельно изучать анти-BPS-решения, так как они превращаются в BPS-решения при умножении $\Phi$ на -1 .

Пусть $\Phi \in \mathcal{M}$ - произвольное решение некоммутативной $U(1)$-модели. Ортопроектор $P: H \rightarrow H$ называется унитоном для $\Phi$ (или $\Phi$-унитоном), если $I-2 P \in \mathcal{M}$ и выполнены уравнения $P \Phi_{-} P^{\perp}=0, P^{\perp} \Phi_{-} P+2 P^{\perp} a P=0$. Тогда унитарный оператор $\Psi=\Phi(I-2 P) \in \mathcal{M}$ также является решением, причем $e(\Psi)=e(\Phi)+$ $Q(P)$ (см. [5], утверждение 9). Операция добавления унитона обратима по формуле $\Phi=-\Psi\left(I-2 P^{\perp}\right)$. Каждое решение $\Phi \in \mathcal{M}$ допускает канонические положительный и отрицательный унитоны $P_{\Phi}^{+}$и $P_{\Phi}^{-}$, заданные формулами $\operatorname{im} P_{\Phi}^{+}=\operatorname{im} \Phi_{+}$и $\operatorname{ker} P_{\Phi}^{-}=\operatorname{im} \Phi_{-}$(см. [5], утверждение 10). Пусть $P: H \rightarrow H-$ некоторый ортопроектор с $\operatorname{dim} \operatorname{im} P<\infty$ и пусть $\Phi \in \mathcal{M}-$ произвольное решение. Если

$$
\operatorname{im} \Phi_{+} \subset \operatorname{im} P, \quad\left(\Phi_{-}+2 a\right)(\operatorname{im} P) \subset \operatorname{im} P,
$$

то $P$ является $\Phi$-унитоном (и называется базовым положительным унитоном). Условия (1.3) часто возникают в дальнейшем (причем всегда для случая, когда $\Phi$ есть BPS-решение), потому что они выполняются для унитона, обратного к каноническому отрицательному унитону (см. [5], п. 3.4).

Важной характеристикой любого решения $\Phi$ является его канонический ранг

$$
r(\Phi):=\operatorname{dimim} \partial_{+} \Phi=\operatorname{dimim} \partial_{-} \Phi=\operatorname{dimim} \Phi_{+}=\operatorname{dimim} \Phi_{-} .
$$

Число $r(\Phi)$ показывает, насколько возрастает (убывает) энергия решения $\Phi$ при добавлении к нему канонического положительного (отрицательного) Ф-унитона. Для любого решения $\Phi \in \mathcal{M}$ ненулевой энергии имеем $1 \leqslant r(\Phi) \leqslant e(\Phi)$, причем крайние случаи $r(\Phi)=1, r(\Phi)=e(\Phi)$ реализуются только в том случае, когда $\Phi$ эквивалентно BPS-решению (см. утверждение 13 работы [5], которое уточняется ниже в замечании 2). Здесь эквивалентность решений $\Phi, \Psi \in \mathcal{M}$ означает, что $\Psi=e^{i \theta} \Phi$ для некоторого $\theta \in \mathbb{R}$ (см. [5], замечание 6). Чтобы зафиксировать выбор представителя в каждом классе эквивалентности, можно перейти от $\mathcal{M}$ к множеству $\mathcal{M}_{0}:=\{\Phi \in \mathcal{M} \mid \operatorname{dim} \operatorname{im}(\Phi-I)<\infty\}$ (т.е. считать $\theta=0$ в определении $\mathcal{M}$ ). Ясно, что $\mathcal{M}_{0}$ и $\mathcal{M}=\mathcal{M}_{0} \times S^{1}$ являются бесконечномерными незамкнутыми подгруппами в унитарной группе $U(H)$ гильбертова пространства $H$.

Определим минимальное унитонное число $u(\Phi)$ любого решения $\Phi \in \mathcal{M}$ как наименьшее $n$, для которого существует разложение $\Phi=\Phi_{0}\left(I-2 P_{1}\right) \ldots\left(I-2 P_{n}\right)$, где $e\left(\Phi_{0}\right)=0$ и каждый ортопроектор $P_{j}$ является унитоном для решения $\Phi_{0}(I-$ $\left.2 P_{1}\right) \ldots\left(I-2 P_{j-1}\right)$ (см. [5], утверждение 11$)$. Из определения унитона вытекает, что $u(\Phi)=1$ тогда и только тогда, когда $\Phi$ эквивалентно BPS-решению (за тривиальным исключением решений с $e(\Phi)=0$ : они эквивалентны BPS-решению $\Phi=I$, но 
имеют $u(\Phi)=0)$. Ясно, что $u(\Phi) \leqslant e(\Phi)$ для любого решения $\Phi$. Поскольку числа $e(\Phi), r(\Phi), u(\Phi)$ не меняются при переходе к эквивалентному решению, мы можем, не теряя общности, рассматривать следующие множества как основные объекты изучения в настоящей работе:

$$
\mathcal{M}(e, r, u):=\left\{\text { все решения } \Phi \in \mathcal{M}_{0} \text { с } e(\Phi)=e, r(\Phi)=r, u(\Phi)=u\right\}
$$

где $e, r, u$ - положительные целые числа, причем $r \leqslant e$ и $u \leqslant e$. В отличие от множеств решений фиксированной энергии $e$, пространства (1.5) в общем случае незамкнуты и часто несвязны в топологии нормы на $U(H)$. Однако во всех изученных ниже случаях пространства $\mathcal{M}(e, r, u)$, если они непусты, допускают взаимно однозначную вещественно-аналитическую параметризацию некоторым алгебраическим многообразием комплексной размерности $r$. Это неожиданное явление вряд ли можно обнаружить стандартным способом, рассматривая ядро линеаризации уравнений Эйлера-Лагранжа. Оно безусловно заслуживает дальнейшего изучения.

1.2. Структура работы. В разделе 2 мы описываем множество всех BPS-решений энергии $е$ как экземпляр $\mathbb{C}^{e}$, вложенный в унитарную группу $U(H)$. Это автоматически дает описание всех решений с $e \leqslant 2, r=1$ или $r=e$ : все они являются BPS-решениями. В разделе 3 доказано, что всякое грассманово решение $G$ дефекта 2 порождает сферу неграссмановых решений, интерполирующую между $G$ и некоторым BPS-решением той же энергии. Поскольку любое не-BPS-решение с $r=e-1$ принадлежит одной из этих сфер, мы автоматически получаем описание всех решений с $e=3$ (раздел 4). В разделе 5 установлено, что множество всех не-BPS-решений энергии $е$ и канонического ранга $r=2$ является прямым произведением $\mathbb{C}^{1}$ на цепочку из $[(e-1) / 2]$ двумерных сфер, последовательно склеенных друг с другом своими полюсами. Это автоматически дает описание случая $e=4$ (раздел 6). В разделе 7 изучены случаи $r=e-2$ (частично) и $e=5$. В качестве приложения найдено число реализуемых нулевых мод гессиана функционала энергии (1.1) вокруг диагональных BPS-решений энергии $e \leqslant 5$ (см. замечание 9). В разделе 8 мы вкратце суммируем полученные результаты и формулируем ряд гипотез.

\section{2. ВРS-РЕШЕНИЯ}

Чтобы построить инвариантные подпространства оператора $A$, можно использовать ядра операторов $f(A)$ для надлежащих функций $f(z)$. Применим эту идею для описания BPS-решений, т.е. инвариантных подпространств оператора уничтожения $a$. Пусть $\Pi(z)=z^{m}+c_{1} z^{m-1}+\cdots+c_{m}$ есть произвольный монический (т.е. со старшим коэффициентом 1 ) комплекснозначный полином степени $m$. Тогда корректно определены оператор П $(a): D_{\infty} \rightarrow D_{\infty}$ и векторное подпространство $\operatorname{ker} \Pi(a):=\left\{x \in D_{\infty} \mid \Pi(a) x=0\right\}$.

УтВеРЖДЕНИЕ 1. А. Оператор $\Phi=I-2 P \in \mathcal{M}$ является ВРS-решением тогда и только тогда, когда образ $V:=\operatorname{im} P$ ортопроектора $P$ либо совпадает со всем пространством $H$, либо имеет вид $V=\operatorname{ker} \Pi(a)$ для некоторого монического 
полинома $\Pi(z)$. Для каждого $m=1,2, \ldots$ отображение $\Pi \mapsto \operatorname{ker} \Pi(a)$ устанавливает взаимно однозначное соответствие между множеством всех монических полиномов П $(z)$ степени $m$ и множеством всех BPS-решений размерности $m$.

Б. Пусть $\Phi=I-2 P$ есть $B P S$-решение $c \operatorname{im} P=\operatorname{ker} \Pi(a)$. Тогда энергия $е(\Phi)$ равна степени полинома $\Pi(z)$, а канонический ранг $r(\Phi)$ равен числу различных корней полинома $\Pi(z)$.

В. Для $1 \leqslant r \leqslant$ е обозначим через $N(e, r)$ число представлений е в виде суммы $r$ неубывающих положительных иелых слагаемых. Тогда множество $\mathcal{M}(e, r, 1)$ всех BPS-решений энергии е и канонического ранга $r$ является обгединением $N(e, r)$ непересекающихся связных компонент. Каждая компонента допускает вещественно-аналитическую взаимно однозначную параметризацию некоторым открытым подмножеством пространства $\mathbb{C}^{r}$.

Г. Множество $\bigcup_{r=1}^{e} \mathcal{M}(e, r, 1)$ всех BPS-решений энергии е совпадает с образом $\mathbb{C}^{e}$ при некотором вещественно-аналитическом вложении $\mathbb{C}^{e} \rightarrow U(H)$. Прообразы множеств $\mathcal{M}(e, r, 1)$ при этом вложении задают стратификацию пространства $\mathbb{C}^{е}$ комплексными подмногообразиями комплексной размерности $r$. Замыкание каждого множества $\mathcal{M}(e, r, 1)$ равно $\bigcup_{\rho=1}^{r} \mathcal{M}(e, \rho, 1)$.

ДокАЗАТЕльство. А. Чтобы оператор $\Phi=I-2 P$ принадлежал $\mathcal{M}$, пространство $V=\operatorname{im} P$ должно иметь конечную размерность или коразмерность. Заметим, что оператор $a$ не имеет инвариантных подпространств $V \subset H$ конечной коразмерности, кроме $V=H$. Действительно, иначе $V^{\perp}$ было бы конечномерным инвариантным подпространством оператора $a^{*}$. Но всякий линейный оператор на конечномерном пространстве имеет собственный вектор, а $a^{*}$ не имеет собственных векторов в $H$ (что легко вывести из определения $a^{*}$, данного в п. 1.1). Получаем требуемое утверждение.

Б. Для описания конечномерных BPS-решений введем векторы

$$
R_{\lambda}:=\sum_{j=0}^{\infty} \frac{\lambda^{j}}{\sqrt{j !}} e_{j}, \quad R_{\lambda}^{k}:=\left(a^{*}-\bar{\lambda} I\right)^{k} R_{\lambda} \quad \text { для } \quad \lambda \in \mathbb{C}, \quad k=0,1,2, \ldots
$$

Нормированные векторы $R_{\lambda} /\left|R_{\lambda}\right|$ известны как когерентные состояния для гармонического осциллятора (см., например, [7]). Хорошо известно, что любое конечное подмножество системы $\left\{R_{\lambda}^{k} \mid \lambda \in \mathbb{C}, k=0,1,2, \ldots\right\}$ состоит из линейно независимых векторов. Кроме того, прямое вычисление в обозначениях п. 1.1 показывает, что $R_{\lambda}$ есть единственный (с точностью до скалярного множителя) собственный вектор оператора $а$ с собственным значением $\lambda$ :

$$
\operatorname{ker}(a-\lambda I)=\left\langle R_{\lambda}\right\rangle \quad \text { для всех } \lambda \in \mathbb{C} .
$$

Из коммутационной формулы $\left[A, A^{* k}\right]=k\left(A^{*}\right)^{k-1}$, справедливой для оператора $A=$ $a-\lambda I$ при любом $\lambda \in \mathbb{C}$, вытекает, что $(a-\lambda I) R_{\lambda}^{k}=k R_{\lambda}^{k-1}$. Поэтому можно рассматривать $R_{\lambda}^{k}$ как присоединенные векторы к собственному вектору $R_{\lambda}$. По индукции получаем следующее обобщение формулы (2.2):

$$
\operatorname{ker}(a-\lambda I)^{k}=\left\langle R_{\lambda}, R_{\lambda}^{1}, \ldots, R_{\lambda}^{k-1}\right\rangle \quad \text { для } \quad \lambda \in \mathbb{C}, \quad k=0,1,2, \ldots
$$


Кроме того, из той же коммутационной формулы по индукции следует, что базис в правой части равенства (2.3) ортогонален.

Пусть теперь $V \subset D_{\infty}$ - конечномерное инвариантное подпространство оператора $a$. Сужение $a$ на $V$ распадается в прямую сумму жордановых клеток. Их собственные значения различны, поскольку каждое собственное подпространство оператора $a$ одномерно (см. (2.2)). Каждая из клеток однозначно определяется своим собственным значением $\lambda$ и размерностью $k$ по формуле $(2.3)$. Если сужение $a$ на $V$ распадается на $r$ жордановых клеток, имеющих собственные значения $\lambda_{j}$ и размерности $k_{j}, j=1,2, \ldots, r$, то $V=\operatorname{ker} \Pi(a)$, где $\Pi(z)=\left(z-\lambda_{1}\right)^{k_{1}} \ldots\left(z-\lambda_{r}\right)^{k_{r}}$. В частности, размерность $\operatorname{dim} V=k_{1}+\cdots+k_{r}$ равна степени полинома П $(z)$. Обратно, для любого монического полинома $\Pi(z)=\left(z-\lambda_{1}\right)^{k_{1}} \ldots\left(z-\lambda_{r}\right)^{k_{r}}$ (где все $\lambda_{j}$ различны) степени $m=k_{1}+\cdots+k_{r}$ пространство

$$
\operatorname{ker} \Pi(a)=\left\langle R_{\lambda_{j}}^{l_{j}} \mid 1 \leqslant j \leqslant r, 0 \leqslant l_{j} \leqslant k_{j}-1\right\rangle
$$

является $m$-мерным и, очевидно, инвариантным относительно $a$.

Равенство $e(\Phi)=m=\operatorname{deg} \Pi$ доказано. Покажем, что $r(\Phi)=r$. Для этого вычислим значение оператора $\Phi_{+}=2 P^{\perp} a^{*} P$ на каждом базисном векторе (2.4). Получаем, что $\operatorname{im} \Phi_{+}=\left\langle P^{\perp} R_{\lambda_{j}}^{k_{j}} \mid j=1, \ldots, r\right\rangle$. Это пространство $r$-мерно, поскольку каждая конечная система векторов $R_{\lambda}^{k}$ линейно независима.

В. Компонента множества $\mathcal{M}(e, r, 1)$, отвечающая представлению $e=k_{1}+\cdots+k_{r}$, состоит из всех решений вида $(2.4)$ с фиксированными $k_{1}, \ldots, k_{r}$ и произвольными наборами $\xi:=\left(\lambda_{1}, \ldots, \lambda_{r}\right) \in \mathbb{C}^{r} \backslash \Delta_{r}$, где $\Delta_{r}$ - замкнутое $(r-1)$-мерное множество всех точек из $\mathbb{C}^{r}$, имеющих хотя бы две равные координаты. Множество $\mathbb{C}^{r} \backslash \Delta_{r}$ связно, так как вещественная коразмерность множества $\Delta_{r}$ в $\mathbb{C}^{r}$ равна 2 . Поскольку некоторые пространства (2.4) с разными $\xi$ могут совпадать (перестановка корней одинаковой кратности не меняет полинома), иногда необходимо профакторизовать $\mathbb{C}^{r} \backslash \Delta_{r}$ по действию надлежащей конечной группы перестановок. Однако все факторпространства указанного вида снова будут биголоморфны некоторым открытым связным подмножествам в $\mathbb{C}^{r}$, так как монические полиномы можно параметризовать не корнями, а коэффициентами (см. замечание 3 ниже). Наконец, параметризация множества всех подпространств вида (2.4) посредством $\xi \in \mathbb{C}^{r} \backslash \Delta_{r}$ является вещественно-аналитической, потому что каждый вектор $R_{\lambda}^{l}$ зависит от $\lambda$ вещественно-аналитически (и даже голоморфно при $l=0$ ), а ортопроектор на пространство $V$, натянутое на столбцы матрицы $M$, задается формулой $M\left(M^{*} M\right)^{-1} M^{*}$.

Г. Множество $\bigcup_{r=1}^{e} \mathcal{M}(e, r, 1)$ отождествляется с $\mathbb{C}^{e}$, если сопоставить каждому моническому полиному $\Pi(z)=z^{e}+c_{1} z^{e-1}+\cdots+c_{e}$ степени $e$ набор $\left(c_{1}, \ldots, c_{e}\right)$ его коэффициентов. Для доказательства вещественной аналитичности полученного отображения $\mathbb{C}^{e} \rightarrow U(H)$ достаточно построить (хотя бы локально по $c_{1}, \ldots, c_{e}$ ) такой базис векторного пространства $\operatorname{ker} \Pi(a)$, который зависел бы голоморфно от коэффициентов полинома П $(z)$. Это делается выбором надлежащей линейной комбинации векторов $R_{\lambda_{1}}, \ldots, R_{\lambda_{e}}$, образующей базис пространства $\operatorname{ker} \Pi(a)$ даже в том предельном случае, когда некоторые из корней $\lambda_{1}, \ldots, \lambda_{e}$ совпадают (например, при $e=2$ такой базис состоит из векторов $\left.R_{\lambda_{1}},\left(R_{\lambda_{2}}-R_{\lambda_{1}}\right) /\left(\lambda_{2}-\lambda_{1}\right)\right)$. После этого остается применить теорему об устранимой особенности, гласящую, что непрерывная 
функция, голоморфная вне некоторого множества комплексной коразмерности 1 , голоморфна всюду. Утверждения о стратификации пространства $\mathbb{C}^{e}$ и о замыкании множества $\mathcal{M}(e, r, 1)$ становятся тогда очевидными.

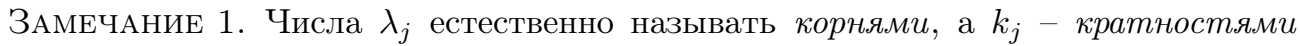
BPS-решения $V=\operatorname{ker} \Pi(a)$, где $\Pi(z)=\left(z-\lambda_{1}\right)^{k_{1}} \ldots\left(z-\lambda_{r}\right)^{k_{r}}$ и все $\lambda_{j}$ различны. В этих терминах результат добавления канонического положительного унитона к любому BPS-решению снова является BPS-решением, полученным из исходного увеличением кратности каждого его корня на единицу (см. доказательство п. В утверждения 1). Аналогично доказывается, что добавление канонического отрицательного унитона к произвольному BPS-решению сводится к уменьшению кратности каждого корня этого решения на единицу.

ЗАмЕчАниЕ 2. Из утверждения 13 работы [5] вытекает, что наибольший (e-мерный) и наименьший (одномерный) страты множества $\bigcup_{r=1}^{e} \mathcal{M}(e, r, 1)$ исчерпывают множества всех (не обязательно BPS) решений $\Phi \in \mathcal{M}_{0}$ с $r=e$ и $r=1$ соответственно. Таким образом, любое решение $\Phi \in \mathcal{M}$ энергии $e(\Phi)=m$ и канонического ранга $r(\Phi)=m$ или $r(\Phi)=1$ эквивалентно BPS-решению, у которого все $m$ корней различны или, наоборот, все $m$ корней совпадают. Из утверждения 14 работы [5] получаем явное описание всех решений $\Phi \in \mathcal{M}_{0}$ энергии $e=1$ или $e=2$ : их множество есть образ $\mathbb{C}^{1}$ или $\mathbb{C}^{2}$ при вложении в унитарную группу $U(H)$, состоящий из отражений относительно ортогональных дополнений к BPS-подпространствам размерности 1 или 2 соответственно.

ЗАмЕчАниЕ 3. Для иллюстрации п. В утверждения 1 рассмотрим первый нетривиальный случай: $e=4, r=2$. Число 4 имеет два допустимых представления: $1+3$ и $2+2$. Поэтому $\mathcal{M}(4,2,1)=\mathcal{M}_{1} \cup \mathcal{M}_{2}$, где $\mathcal{M}_{1}$ состоит из всех BPS-решений $\operatorname{ker} \Pi(a)$ с $\Pi(z)=\left(z-\lambda_{1}\right)\left(z-\lambda_{2}\right)^{3}$, а $\mathcal{M}_{2}$ - из всех BPS-решений $\operatorname{ker} \Pi(a)$ с $\Pi(z)=$ $\left(z-\lambda_{1}\right)^{2}\left(z-\lambda_{2}\right)^{2}$. Во втором случае пространство $\operatorname{ker} \Pi(a)$ не меняется при перестановке $\lambda_{1}$ и $\lambda_{2}$. Поэтому $\mathcal{M}_{1} \cong \mathbb{C}^{2} \backslash \Delta_{2}$, но $\mathcal{M}_{2} \cong\left(\mathbb{C}^{2} \backslash \Delta_{2}\right) / S_{2}$, где $S_{2}$ есть группа перестановок двух координат. Однако отображение $\left(\lambda_{1}, \lambda_{2}\right) \mapsto\left(\mu_{1}, \mu_{2}\right)$, заданное формулами $\mu_{1}=\lambda_{1}+\lambda_{2}$ и $\mu_{2}=\lambda_{1} \lambda_{2}$, биголоморфно отождествляет факторпространство $\mathcal{M}_{2} \cong\left(\mathbb{C}^{2} \backslash \Delta_{2}\right) / S_{2}$ с открытым связным подмножеством $\left\{\mu \in \mathbb{C}^{2} \mid \mu_{1}^{2}-4 \mu_{2} \neq 0\right\}$ пространства $\mathbb{C}^{2}$.

ЗАмечАниЕ 4. Кроме конечномерных инвариантных подпространств, оператор уничтожения $а$ имеет замкнутые инвариантные подпространства $V \subset H$ бесконечной размерности и коразмерности: $V=\operatorname{ker} f(a)$ для неполиномиальных целых функций $f(z)$ надлежащего роста (грубо говоря, $\ln |f(z)| \leqslant C|z|^{2}$; см. подробности в работе [8] или в приложении 1 книги [7]). Например, если $f(z)=\sin z$, то $V$ есть замыкание линейной оболочки всех векторов $R_{\lambda}$ с $\lambda \in \mathbb{Z}$. Хотя ортопроекторы $P$ с образом $\operatorname{im} P=V$ указанного вида не удовлетворяют условию $I-2 P \in \mathcal{M}$, для них выполнено BPS-условие $P^{\perp} a P=0$ на всюду плотном подпространстве в $H$. Если $f$ - экспоненциальный полином, то оператор $[a, \Phi]($ с $\Phi=I-2 P$ и im $P=\operatorname{ker} f(a))$ плотно определен на $H$, но не продолжается до оператора Гильберта-Шмидта на всем $H$ (см. [9]). Поэтому пространства $V=\operatorname{ker} f(a)$ можно интерпретировать как 
"BPS-решения бесконечной энергии". Их коммутативные аналоги - это голоморфные нерациональные отображения из $\mathbb{C}$ в грассманианы $\operatorname{Gr}_{k}\left(\mathbb{C}^{n}\right) \subset U(n)$.

\section{3. ГРАССМАНОВЫ РЕШЕНИЯ ДЕФЕКТА 2 И СФЕРЫ НЕГРАССМАНОВЫХ РЕШЕНИЙ}

Известно ([5], утверждение 15), что всякое грассманово решение энергии $е$ и дефекта 2 представляет собой $(e-2)$-мерное подпространство вида $G:=M \ominus S$, где $M$ и $S$ суть BPS-решения энергии $e-1$ и 1 соответственно, причем $S \subset M$ и $a^{*} S \subset M$; здесь и далее через $\ominus$ обозначается ортогональное дополнение. Опишем эту геометрическую ситуацию более подробно. Прежде всего, из утверждения 1 вытекает, что $S=\left\langle R_{\lambda}\right\rangle$ для некоторого $\lambda \in \mathbb{C}$. Для упрощения обозначений мы заменим все рассматриваемые унитарные операторы $\Phi$ на $U_{\lambda} \Phi U_{\lambda}^{*}$, где $U_{\lambda}$ есть некоммутативный сдвиг ${ }^{1)}$ на $\lambda$. Это позволяет нам считать, что $\lambda=0$ и, следовательно, $S=\left\langle R_{0}\right\rangle=\left\langle e_{0}\right\rangle$. Пусть $k$ - наибольшее целое число такое, что $e_{0}, e_{1}, \ldots, e_{k-1} \in M$. Условия $S \subset M$ и $a^{*} S \subset M$ эквивалентны неравенству $k \geqslant 2$. Введем еще одно BPS-подпространство $B:=M \oplus\left\langle e_{k}\right\rangle$ размерности $e$ (здесь и далее через $\oplus$ обозначается прямая сумма векторных подпространств, не обязательно ортогональная). Обозначим через $P_{0}$ ортопроектор в $H \mathrm{c} \operatorname{im} P_{0}=\left\langle e_{0}\right\rangle$, а через $P(c)$ - ортопроектор в $H$ с $\operatorname{im} P(c)=V(c):=G \oplus\left\langle e_{0}+c e_{k}\right\rangle \subset B$, где $c \in \mathbb{C}^{1}-$ любое фиксированное число. Эти обозначения сохраняют смысл и в предельном случае $c=\infty$, когда $\operatorname{im} P(\infty)=V(\infty)=G \oplus\left\langle e_{k}\right\rangle=B \ominus\left\langle e_{0}\right\rangle$. Рассмотрим семейство унитарных операторов

$$
\Phi(c):=\left(I-2 P_{0}\right)(I-2 P(c)) \in \mathcal{M}_{0}, \quad c \in \mathbb{C} P^{1} .
$$

Оно представляет собой сферу $\mathbb{C} P^{1}$, вложенную в унитарную группу $U(H)$ и интерполирующую между грассмановым решением $\Phi(0)=I-2 P_{G}$ дефекта 2 и BPSрешением $\Phi(\infty)=I-2 P_{B}$, где $P_{G}$ и $P_{B}$ - ортопроекторы с образами $G$ и $B$ соответственно.

УтВЕРЖДЕНИЕ 2. А. Все операторы (3.1) являются решениями энергии $e(\Phi(c))=е$ с унитонным числом $u(\Phi(c))=2$, кроме $u(\Phi(\infty))=1$.

Б. Канонический ранг любого решения (3.1) для $c \neq \infty$ равен $r(\Phi(c))=$ $r(\Phi(\infty))+1$.

В. C точностью до некоммутативного сдвига всякое не-BPS-решение $\Phi \in \mathcal{M}_{0}$ с е $(\Phi)=$ е и $r(\Phi)=e-1$ принадлежит некоторой сфере (3.1).

ДоказАтельство. А. Покажем, что каждый ортопроектор $P(c)$ удовлетворяет условиям (1.3) для $\Phi=\Phi^{0}:=I-2 P_{0}$ и, следовательно, является $\Phi^{0}$-унитоном. Действительно, поскольку $k \geqslant 2$, имеем $V(c) \supset G \supset\left\langle e_{1}\right\rangle=\operatorname{im} \Phi_{+}^{0}$, и первое условие (1.3)

\footnotetext{
1) Иными словами, $U_{\lambda}$ - это единственный унитарный оператор на $H$, переводящий каждое когерентное состояние $R_{\alpha} /\left|R_{\alpha}\right|$ в $R_{\alpha+\lambda} /\left|R_{\alpha+\lambda}\right|$ (см. [7]). Поскольку $U_{\lambda} a U_{\lambda}^{*}=a-\lambda I$, сопряжение унитарных операторов посредством $U_{\lambda}$ не меняет функционал энергии (1.1) и отображает решения $U(1)$-модели снова в решения той же энергии и того же канонического ранга (см., например, [4], п. 2.2). Оно также перестановочно со всеми конструкциями теории унитонов ([5], п. 3.2, 3.3). Коммутативный аналог этого преобразования заменяет $\varphi(z)$ на $\varphi(z-\lambda)$ в обозначениях работы [5] (п. 1.1).
} 
выполнено. С другой стороны, формула (3.12) работы [5] и очевидное равенство $a P_{0}=0$ показывают, что $\Phi_{-}^{0}+2 a=2 P_{0}^{\perp} a$. Поэтому второе условие (1.3) принимает вид $P_{0}^{\perp} a(V(c)) \subset V(c)$. Оно выполнено, поскольку $P_{0}^{\perp} a(V(c)) \subset P_{0}^{\perp} a B=$ $P_{0}^{\perp} M=G \subset V(c)$. Итак, все операторы (3.1) являются решениями. Их энергия равна $e(\Phi(c))=e\left(\Phi^{0}\right)+Q(P(c))=1+(e-1)=e$ (см. [5], п. Б утверждения 9). Их унитонное число не превосходит 2 по определению и равно 2 при $c \neq \infty$, так как в этом случае $\Phi(c)$ не являются BPS-решениями.

Б. Покажем, что оператор $\Phi(c)_{+}$равен нулю на ортогональном дополнении $B^{\perp}$ пространства B. Действительно, согласно п. А утверждения 9 работы [5] имеем

$$
\Phi(c)_{+}=\Phi_{+}^{0}+2\left[a^{*}, P(c)\right] .
$$

Если $x \in B^{\perp}$, то $P_{0} x=0$, так что оператор $\Phi_{+}^{0}=2 P_{0}^{\perp} a^{*} P_{0}$ обращается в нуль на векторе $x$. С другой стороны, если $x \in B^{\perp}$, то $a^{*} x \in B^{\perp}$ в силу инвариантности $B$ относительно $a$. Отсюда $\left[a^{*}, P(c)\right] x=a^{*} P(c) x-P(c) a^{*} x=a^{*} 0-0=0$, что и требовалось показать. Чтобы вычислить действие $\Phi(c)_{+}$на $B$, рассмотрим единичный вектор нормали $n=n(c)$ к гиперплоскости $V(c) \subset B$ и запишем базис (2.4) пространства $B$ в виде

$$
\begin{aligned}
B= & \left\langle e_{0}, e_{1}, \ldots, e_{k} ; R_{\alpha_{2}}, R_{\alpha_{2}}^{1}, \ldots, R_{\alpha_{2}}^{k_{2}-1} ; \ldots ; R_{\alpha_{s}}, R_{\alpha_{s}}^{1}, \ldots, R_{\alpha_{s}}^{k_{s}-1}\right\rangle= \\
= & \langle n\rangle \oplus V(c)=\left\langle n ; e_{0}+c e_{k}, e_{1}, \ldots, e_{k-1} ; P_{0}^{\perp} R_{\alpha_{2}}, P_{0}^{\perp} R_{\alpha_{2}}^{1}, \ldots, P_{0}^{\perp} R_{\alpha_{2}}^{k_{2}-1} ; \ldots ;\right. \\
& \left.P_{0}^{\perp} R_{\alpha_{s}}, P_{0}^{\perp} R_{\alpha_{s}}^{1}, \ldots, P_{0}^{\perp} R_{\alpha_{s}}^{k_{s}-1}\right\rangle,
\end{aligned}
$$

где $\alpha_{2}, \ldots, \alpha_{s} \in \mathbb{C} \backslash\{0\}, \alpha_{i} \neq \alpha_{j}$ при $i \neq j$. Здесь число $s \geqslant 1$ равно каноническому рангу $r(\Phi(\infty))$ согласно п. В утверждения 1 , причем в случае $s=1$ равенство (3.3) упрощается до $B=\left\langle e_{0}, e_{1}, \ldots, e_{k}\right\rangle=\left\langle n ; e_{0}+c e_{k}, e_{1}, \ldots, e_{k-1}\right\rangle$. Из формулы $P(c) x=$ $x-(x, n) n$, верной для всех $x \in B$, легко получить, что

$$
\left[a^{*}, P(c)\right] x=P_{B}^{\perp} a^{*} x+\left(a^{*} x, n\right) n-(x, n) a^{*} n \quad \text { для всех } \quad x \in B .
$$

Слагаемое $(x, n) a^{*} n$ обращается в нуль на всех векторах $x$ второго базиса (3.3), кроме $x=n$. Если же $x=n$, то обе части формулы (3.4) равны $-P_{V(c)} a^{*} n$, откуда $\Phi(c)_{+} n=2\left(n, e_{0}\right) e_{1}-2 P_{V(c)} a^{*} n=0$ (первое равенство есть не что иное, как $(3.2)$, а второе вытекает из того, что векторы $a^{*} n$ и $\left(n, e_{0}\right) e_{1}$ имеют одинаковые скалярные произведения со всеми перечисленными в (3.3) базисными векторами пространства $V(c))$. Пользуясь $(3.2),(3.4)$ и очевидной формулой $P_{0}^{\perp} R_{\alpha_{j}}^{l}=R_{\alpha_{j}}^{l}-\left(-\bar{\alpha}_{j}\right)^{l} e_{0}$, мы получаем

$$
\begin{aligned}
\Phi(c)_{+}\left(e_{0}+c e_{k}\right) & =2 e_{1}+2 c P_{B}^{\perp} a^{*} e_{k}+2 c\left(a^{*} e_{k}, n\right) n, \\
\Phi(c)_{+}\left(e_{k-1}\right) & =2\left(a^{*} e_{k-1}, n\right) n, \\
\Phi(c)_{+}\left(P_{0}^{\perp} R_{\alpha_{j}}^{k_{j}-1}\right) & =2 P_{B}^{\perp} a^{*} R_{\alpha_{j}}^{k_{j}-1}+2\left(a^{*} R_{\alpha_{j}}^{k_{j}-1}, n\right) n, \quad 2 \leqslant j \leqslant s .
\end{aligned}
$$

Действие оператора $\Phi(c)+$ на остальных векторах $x$ второго базиса (3.3) равно нулю: для этих векторов имеем $x \perp e_{0}, x \in V(c)$ и $a^{*} x \in V(c)$, так что оба слагаемых в правой части (3.2) обращаются в нуль на $x$. Заметим, что вектор $\Phi(c)_{+}\left(e_{k-1}\right)$ яв- 
ляется ненулевым кратным вектора $n^{2)}$. Поэтому пространство $\mathrm{im} \Phi(c)_{+}$является линейной оболочкой вектора $n$ и $s$ векторов $e_{1}+c P_{B}^{\perp} a^{*} e_{k}, P_{B}^{\perp} a^{*} R_{\alpha_{j}}^{k_{j}-1}, 2 \leqslant j \leqslant s$, ортогональных к $n$. Эти $s$ векторов линейно независимы в силу такого же рассуждения, как в доказательстве п. Б утверждения 1. Тем самым $r(\Phi(c))=s+1$.

В. Добавляя к $\Phi$ канонический отрицательный $\Phi$-унитон, получим некоторое решение $\Phi^{0}$ энергии $e=1$. С точностью до некоммутативного сдвига и эквивалентности можно считать, что $\Phi^{0}=I-2 P_{0}$, где im $P_{0}=\left\langle e_{0}\right\rangle$ (см. утверждение 1 или замечание 2). Согласно п. А утверждения 13 работы [5] достаточно показать, что всякий положительный базовый $\Phi^{0}$-унитон $P$ ранга $e-1$ совпадает с $P(c)$ для некоторого $c \in \mathbb{C}$, если только $\Phi$ не является BPS-решением. Иными словами, надо описать все подпространства $V:=\operatorname{im} P$ размерности $e-1$, удовлетворяющие условиям (1.3) для $\Phi=\Phi^{0}$ :

$$
\left\langle e_{1}\right\rangle \subset V, \quad P_{0}^{\perp} a V \subset V .
$$

Если $V$ содержит $e_{0}$, то из условий (3.5) вытекает, что

$$
\left\langle e_{0}, e_{1}\right\rangle \subset V, \quad a V \subset P_{0} a V+P_{0}^{\perp} a V \subset\left\langle e_{0}\right\rangle+P_{0}^{\perp} a V \subset V .
$$

Поэтому $V$ является BPS-решением, содержащим $\left\langle e_{0}, e_{1}\right\rangle$, а $\Phi=\Phi^{0}(I-2 P)$ - грассмановым решением $V \ominus\left\langle e_{0}\right\rangle$ дефекта 2. Такое решение $\Phi$ принадлежит одной из сфер (3.1) согласно утверждению 15 работы [5].

Таким образом, можно считать, что $e_{0} \notin V$. Тогда

$$
a\left(\left\langle e_{0}\right\rangle \oplus V\right)=a V \subset P_{0} a V+P_{0}^{\perp} a V \subset\left\langle e_{0}\right\rangle+P_{0}^{\perp} a V \subset\left\langle e_{0}\right\rangle \oplus V
$$

в силу второго условия (3.5). Следовательно, $B:=\left\langle e_{0}\right\rangle \oplus V$ является BPS-подпространством, содержащим $e_{0}$ (по определению) и $e_{1}$ (в силу первого условия (3.5)). Пусть $k$ - максимальное целое число, для которого $e_{0}, e_{1}, \ldots, e_{k} \in B$. Если $k=1$, то первое равенство (3.3) показывает, что $P_{0}^{\perp} B=\left\langle e_{0}\right\rangle \oplus P_{0}^{\perp} a B$. Из очевидного равенства $a B=a V$ и условий (3.5) вытекает, что $P_{0}^{\perp} B \subset V$. Это включение должно быть равенством, поскольку $\operatorname{dim} P_{0}^{\perp} B=\operatorname{dim} V=e-1$. Но тогда $V \perp e_{0}$, так что $\Phi=\Phi^{0}(I-2 P)$ есть BPS-решение вопреки предположению. Таким образом, можно считать, что $k \geqslant 2$. Из первого равенства (3.3) мы опять видим, что второе условие (3.5) эквивалентно включению $G \subset V$, где $G:=P_{0}^{\perp} a B=P_{0}^{\perp} a V$ - соответствующее грассманово решение дефекта 2 . Поскольку $\operatorname{dim} G=e-2$ и $\operatorname{dim} V=e-1$, имеем $V=G \oplus\langle u\rangle$ для некоторого вектора $u \neq 0$ из подпространства $\left\langle e_{0}, e_{k}\right\rangle \subset B$ (это подпространство трансверсально к $G$ ). Записав $u$ в виде $u=e_{0}+c e_{k}$ для некоторого $c \in \mathbb{C} \backslash\{0\}$ (случаи $c=0$ и $c=\infty$ исключаются сделанными выше предположениями), мы видим, что $V=V(c)$ и $\Phi=\Phi(c)$ в обозначениях (3.1).

Чтобы наглядно представить действие унитарных операторов (3.1) на $H$, запишем эти операторы в виде матриц в надлежащем ортонормированном базисе пространства $H$. Приводимая ниже формула (3.6) задает взаимно однозначную параметризацию семейства $(3.1)$ сферой $S^{2}=\left\{(\kappa, \lambda) \in \mathbb{R}^{1} \times\left.\mathbb{C}^{1}\left|\kappa^{2}+\right| \lambda\right|^{2}=1\right\}$.

\footnotetext{
2) Действительно, если бы выполнялось равенство $\left(a^{*} e_{k-1}, n\right)=0$, то мы имели бы $e_{k} \in V(c)$ и, следовательно, $e_{0} \in V(c)$. Но тогда $V(c)=M$, откуда $e_{k} \in M$ вопреки определению $k$.
} 
УтВЕРЖДЕНИЕ 3. Каждый оператор (3.1) имеет инвариантные подпространства $B^{\perp}, G$ и $B \ominus G$, ортогональная прямая сумма которых равна всему пространству Н. При каждом $c \in \mathbb{C} P^{1}$ имеем $\Phi(c) x=x$ для $x \in B^{\perp} u \Phi(c) x=-x$ для $x \in G$. Рассмотрим вектор $N:=P_{G}^{\perp} e_{k}=P_{M}^{\perp} e_{k}$. Тогда матрица унитарного оператора $\Phi(c)$ в ортонормированном базисе $\left\langle e_{0}, N /|N|\right\rangle$ пространства $B \ominus G$ имеет вид

$$
\left.\Phi(c)\right|_{B \ominus G}=\left(\begin{array}{cc}
\kappa & -\bar{\lambda} \\
\lambda & \kappa
\end{array}\right), \quad \kappa=\frac{1-|c|^{2}|N|^{2}}{1+|c|^{2}|N|^{2}}, \quad \lambda=-\frac{2 c|N|}{1+|c|^{2}|N|^{2}}
$$

ДокАЗАтЕЛЬСтво. Формулы для $\left.\Phi(c)\right|_{B^{\perp}}$ и $\left.\Phi(c)\right|_{G}$ вытекают из (3.1). Докажем (3.6). Заметим, что $N \neq 0$ по определению $k$. Поскольку векторы $e_{0}, N$ ортогональны к $G$, их образы при $P(c)$ параллельны вектору нормали к $G$ в пространстве $\operatorname{im} P(c)=G \oplus\left\langle e_{0}+c e_{k}\right\rangle$. Указанный вектор нормали равен $e_{0}+c N$. Записывая $P(c) x=\mu\left(e_{0}+c N\right)$ для $x=e_{0}, N$, мы легко находим из условия $x-P(c) x \perp e_{0}+c N$, что $\mu=\left(1+|c|^{2}|N|^{2}\right)^{-1}$ для $x=e_{0}$ и $\mu=\bar{c}|N|^{2}\left(1+|c|^{2}|N|^{2}\right)^{-1}$ для $x=N$. Подставляя эти результаты в (3.1), получаем (3.6).

ЗАмечАниЕ 5. Из утверждения 2 вытекает, что $\mathcal{M}(e, e-1, u)=\varnothing$ при $u>2$, а $\mathcal{M}(e, e-1,2)$ представляется в виде $D^{e-2} \times \mathbb{C}_{c}^{1}$, где $D^{e-2} \subset \mathbb{C}^{e-2}$ - открытое подмножество, параметризующее все BPS-решения энергии $е$ с кратностями $3,1, \ldots, 1$ (это одна из двух упомянутых в п. В утверждения 1 связных компонент множества $\mathcal{M}(e, e-2,1))$, а множество $\mathbb{C}_{c}^{1}$ получено из сферы $(3.1)$ удалением точки $c=\infty$. Если $e \leqslant 5$, то замыкание множества $\mathcal{M}(e, e-1,2)$ равно $M^{e-2} \times \mathbb{C} P_{c}^{1}$, где $M^{e-2} \subset \mathbb{C}^{e-}$ множество всех BPS-решений, имеющих хотя бы один корень кратности, больше или равной 3. Для произвольных $е$ это замыкание равно объединению сфер (3.1) по всем грассмановым решениям $G$ энергии $e$ и дефекта 2 , причем множество всех таких $G$ слегка отличается от $M^{e-2}$ при $e \geqslant 6$. Заметим еще, что утверждение 2 дает примеры не-BPS-решений (как грассмановых, так и неграссмановых) с любым допустимым значением канонического ранга: $2 \leqslant r \leqslant e-1$ (сравните с замечанием 2 для случаев $r=1$ и $r=e$ ).

\section{4. РЕШЕНИЯ C $e=3$}

Согласно замечанию 2 для всякого не-BPS-решения $\Phi \in \mathcal{M}$ энергии $e(\Phi)=3$ имеем $r(\Phi)=2$. Поэтому п. В утверждения 2 показывает, что $\Phi$ принадлежит (с точностью до некоммутативного сдвига и эквивалентности) сфере (3.1), отвечающей решениям $G=\left\langle e_{1}\right\rangle$ и $B=\left\langle e_{0}, e_{1}, e_{2}\right\rangle$, а утверждение 3 (в котором в этом случае $\left.N=e_{2}\right)$ дает для $\Phi$ следующую матрицу в исходном базисе $\left\{e_{0}, e_{1}, \ldots\right\}$ :

$$
\Phi=\left(\begin{array}{cccc}
k & 0 & -\bar{l} & 0 \\
0 & -1 & 0 & 0 \\
l & 0 & k & 0 \\
0 & 0 & 0 & I_{\infty}
\end{array}\right), \quad k:=\frac{1-|c|^{2}}{1+|c|^{2}}, \quad l:=-\frac{2 c}{1+|c|^{2}}
$$

где $I_{\infty}$ означает единичную матрицу бесконечного ранга. Эти рассуждения доказывают следующее утверждение. 
УтВЕРЖДЕНИЕ 4. С точностъю до некоммутативного сдвига и эквивалентности любое не-BPS-решение $\Phi \in \mathcal{M}$ энергии $е(\Phi)=3$ задается формулой (4.1) для некоторого с $\in \mathbb{C}$. Обратно, для любого $с \in \mathbb{C}$ бормула (4.1) задает не-BPS-решение c $e(\Phi)=3$ ur $(\Phi)=2$.

Таким образом, имеем $\mathcal{M}(3,2,2) \cong \mathbb{C}^{2}=\mathbb{C}_{c}^{1} \times \mathbb{C}_{\lambda}^{1}$, где $\lambda$ параметризует некоммутативные сдвиги. Можно описать пространство

$$
\mathcal{M}(3):=\mathcal{M}(3,3,1) \cup \mathcal{M}(3,2,1) \cup \mathcal{M}(3,1,1) \cup \mathcal{M}(3,2,2) \cong \mathbb{C}^{3} \cup \mathbb{C}^{2}
$$

всех решений $\Phi$ с $e(\Phi)=3$ еще и другим способом: это образ $\mathbb{C}^{3} \cup\left(\mathbb{C} P^{1} \times \mathbb{C}^{1}\right)$ при вещественно-аналитическом вложении в $U(H)$, причем $\mathbb{C}^{3}$ состоит из всех BPS-решений, а $\mathbb{C} P^{1} \times \mathbb{C}^{1}-$ из всех решений, получаемых добавлением двумерных базовых унитонов к BPS-решениям энергии 1. Компоненты $\mathbb{C}^{3}$ и $\mathbb{C} P^{1} \times \mathbb{C}$ рассматриваемого пространства модулей являются гладкими, замкнутыми и неприводимыми. Их пересечение равно множеству всех BPS-решений с $e=3$ и $r=1$. Заметим, что пространства $\mathcal{M}(3, r, u)$ образуют стратификацию множества $\mathcal{M}(3)$ подобно п. Г утверждения 1.

\section{5. РЕШЕНИЯ С $r=2$}

Конструкцию решений (4.1) можно обобщить следующим образом. Для любых целых чисел $s, t$ при $0 \leqslant s \leqslant t-2$ рассмотрим двумерную сферу $S_{s t}^{2}$, состоящую из всех унитарных операторов вида

$$
\Phi=\left(\begin{array}{ccccc}
I_{s} & 0 & 0 & 0 & 0 \\
0 & k & 0 & -\bar{l} & 0 \\
0 & 0 & -I_{t-s-1} & 0 & 0 \\
0 & l & 0 & k & 0 \\
0 & 0 & 0 & 0 & I_{\infty}
\end{array}\right), \quad k \in \mathbb{R}, \quad l \in \mathbb{C}, \quad k^{2}+|l|^{2}=1
$$

где $I_{s}, I_{t-s-1}$ и $I_{\infty}$ - единичные матрицы ранга $s, t-s-1$ и $\infty$ соответственно. Заменяя пару $(k, l)$ на один комплексный параметр $c \in \mathbb{C}$ по формулам (4.1), можно также рассматривать $S_{s t}^{2}$ как комплексную проективную прямую $\mathbb{C} P^{1}$.

УтВЕРЖДЕНИЕ 5. А. Все операторы (5.1) являются решениями с $е(\Phi)=s+t+1$, $r(\Phi)=2$ и $u(\Phi)=2$, кроме случая $s=0, k=-1$, когда $u(\Phi)=r(\Phi)=1$.

Б. Добавление канонического положительного унитона ко всем решениям класca $S_{s t}^{2}$ задает голоморфное (дробно-линейное) взаимно однозначное отображение сферы $S_{s t}^{2}$ на $S_{s+1, t+1}^{2}$. Добавление канонического отрицательного унитона ко всем решениям класса $S_{s+1, t+1}^{2}$ задает обратное отображсение сберы $S_{s+1, t+1}^{2}$ на $S_{s t}^{2}$. Добавляя канонический отрищательный унитон к любому решению класса $S_{0 t}^{2}$, получаем диагональное BPS-решение $\left\langle e_{0}, e_{1}, \ldots, e_{t-2}\right\rangle$ энергии $t-1$.

В. С точностью до некоммутативного сдвига и эквивалентности любое не-ВРSрешение $\Phi \in \mathcal{M}$ с $r(\Phi)=2$ имеет вид (5.1) для некоторых иельх $s, t$ при $0 \leqslant s \leqslant t-2$ и некоторой парь $(k, l) \in \mathbb{R} \times \mathbb{C}$ nри $k^{2}+|l|^{2}=1$. 
ДоказАтельство. А. Можно явно вычислить действие оператора $\Phi_{+}=a^{*}-$ $\Phi^{*} a^{*} \Phi$ на всех векторах стандартного базиса. А именно, если $j \neq s-1, s, t-1, t$, то $\Phi e_{j}= \pm e_{j}$ и $\Phi^{*} e_{j+1}= \pm \Phi^{*} e_{j+1}$, откуда $\Phi_{+} e_{j}=0$. В оставшихся случаях имеем

$$
\begin{array}{ll}
\Phi_{+} e_{s-1}=\sqrt{s}\left((1-k) e_{s}+l e_{t}\right), & \Phi_{+} e_{s}=(1+k) \sqrt{s+1} e_{s+1}-l \sqrt{t+1} e_{t+1}, \\
\Phi_{+} e_{t-1}=\sqrt{t}\left(\bar{l} e_{s}+(1+k) e_{t}\right), & \Phi_{+} e_{t}=-\bar{l} \sqrt{s+1} e_{s+1}+(1-k) \sqrt{t+1} e_{t+1} .
\end{array}
$$

Отсюда прямой проверкой получается, что $\Phi$ является решением (см. [5], п. Б утверждения 3) и $e(\Phi)=s+t+1$ (см. (1.1)). Кроме того, векторы $\Phi_{+} e_{s-1}, \Phi_{+} e_{t-1}$ пропорциональны друг другу (то же самое верно для пары $\Phi_{+} e_{s}, \Phi_{+} e_{t}$ ) и отличны от нуля, за исключением случая $s=0$ и диагонального случая, когда $k= \pm 1, l=0$. Поэтому $\operatorname{im} \Phi_{+}=\left\langle v_{1}, v_{2}\right\rangle$, где ненулевые векторы

$$
v_{1}:=(1-k) e_{s}+l e_{t}, \quad v_{2}:=(1+k) \sqrt{s+1} e_{s+1}-l \sqrt{t+1} e_{t+1}
$$

ортогональны друг другу и, следовательно, линейно независимы. Таким образом, $r(\Phi)=\operatorname{dimim} \Phi_{+}=2$. В п. Б утверждения 2 дается тот же результат в исключительном случае $s=0$ (кроме значения $k=-1$, когда $\Phi$ является BPS-решением). В исключительных случаях $k= \pm 1, l=0, s \neq 0$ тот же самый результат получается небольшой модификацией приведенного рассуждения (обозначим через $v_{1}$ единственный ненулевой вектор из двух векторов $\Phi_{+} e_{s-1}, \Phi_{+} e_{t-1}$, и аналогично для $\left.v_{2}\right)$. Для доказательства равенства $u(\Phi)=2$ заметим, что $\Phi$ получается из диагонального BPS-решения $\Phi_{0}=I-2 P_{0} \mathrm{c} \operatorname{im} P_{0}=\left\langle e_{0}, \ldots, e_{s}\right\rangle$ добавлением положительного

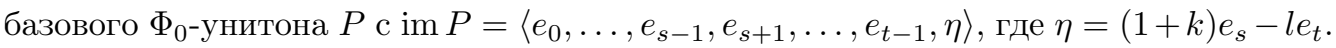
Действительно, условия (1.3) с заменой $\Phi$ на $\Phi_{0}$ легко проверяются явным вычислением (в комплексной координате $c$, вектор $\eta$ пропорционален $e_{s}+c e_{t}$ при $k \neq-1$ ). Отсюда также получается другое доказательство того, что $\Phi$ является решением и $e(\Phi)=s+t+1$.

Б. Пусть $P$ есть канонический положительный $\Phi$-унитон, т.е. $\operatorname{im} P=\operatorname{im} \Phi_{+}$. Действие оператора $I-2 P$ на всех базисных векторах легко найти с помощью (5.2), например

$$
(I-2 P) e_{s}=e_{s}-2 \frac{\left(e_{s}, v_{1}\right)}{\left(v_{1}, v_{1}\right)} v_{1}=k e_{s}-l e_{t} .
$$

Умножая полученные результаты на (5.1), мы видим, что новое решение $\Psi:=$ $\Phi(I-2 P)$ опять имеет вид $(5.1)$ с заменой целых чисел $(s, t)$ на $(s+1, t+1)$, а параметров $(k, l)$ на дробно-линейные выражения ${ }^{3)}$

$$
k^{\prime}=\frac{(s+t+2) k+(s-t)}{(s-t) k+(s+t+2)}, \quad l^{\prime}=\frac{-2 l \sqrt{(s+1)(t+1)}}{(s-t) k+(s+t+2)} .
$$

Таким образом, доказано утверждение о канонических положительных унитонах. Унитон, обратный к каноническому положительному, является базовым согласно п. А утверждения 13 работы [5]. Канонический ранг полученного нового решения

\footnotetext{
3) Зависимость комплексной координаты $c^{\prime}$ от $c$ оказывается даже линейной.
} 
не превосходит 2 ([5], п. Б утверждения 13) и не равен 1 (согласно замечаниям 2 и 1). Следовательно, этот канонический ранг $r=2$, а рассматриваемый базовый унитон является каноническим. Этим доказано утверждение о канонических отрицательных унитонах для $\Phi \in S_{s+1, t+1}^{2}$. Утверждение о канонических отрицательных унитонах для $\Phi \in S_{0 t}^{2}$ можно проверить напрямую с помощью явной формулы для $\Phi_{+}=-\Phi_{-}^{*}$ из доказательства п. А или вывести из п. В, доказательство которого не использует интересующий нас результат.

В. Для доказательства нам потребуется следующая

ЛЕмма. Если $P$ - положителъный базовый унитон ранга $r=2$ для диагонального BPS-решения $\Phi_{0}=I-2 P_{0} c \operatorname{im} P_{0}=\left\langle e_{0}, \ldots, e_{p-1}\right\rangle$, то либо $\Phi_{0}(I-2 P)$ является $B P S$-решением, либо $\operatorname{im} P=\left\langle e_{p}, e_{0}+c e_{p+1}\right\rangle$ для некоторого $c \in \mathbb{C}$.

Действительно, поскольку $\operatorname{im}\left(\Phi_{0}\right)_{+}=\left\langle e_{p}\right\rangle$, мы можем записать $\operatorname{im} P=\left\langle e_{p}, \eta\right\rangle$, где $\eta \perp e_{p}$. Оператор $\left(\Phi_{0}\right)_{-}+2 a$ равен $2 a$ на $\left\langle e_{p}\right\rangle^{\perp}$ и обращается в нуль на векторе $e_{p}$. Поэтому инвариантность $\operatorname{im} P$ относительно $\left(\Phi_{0}\right)_{-}+2 a$ эквивалентна существованию таких $A, B \in \mathbb{C}$, что $2 a \eta=A e_{p}+B \eta$. Если $B \neq 0$, то сравнение проекций обеих частей равенства на $e_{p-1}$ дает $\eta_{p-1}=0$. Тогда сравнение проекций на $e_{p-2}$ дает $\eta_{p-2}=0$ и т.д. В итоге получаем $P_{0} \eta=0$, так что новое решение $\Phi_{0}(I-2 P)$ оказывается BPS-решением. Если же $B=0$, то $2 a \eta=A e_{k}$ и, следовательно, вектор $\eta$ пропорционален $e_{0}+c e_{p+1}$ для некоторого $c \in \mathbb{C}$. Лемма доказана.

Теперь докажем утверждение п. В индукцией по значению энергии $e(\Phi)$, отдельно для четных и нечетных значений. Например, пусть число $e(\Phi)=2 j+1$ нечетно. При $j=0$ утверждение верно, так как все решения энергии $e(\Phi)=1$ являются BPS-решениями. Допустим, что оно верно при всех $j \leqslant j_{0}-1$ и рассмотрим любое не-BPS-решение $\Phi$ с $e(\Phi)=2 j_{0}+1$ и $r(\Phi)=2$. Добавляя к $\Phi$ канонический отрицательный унитон, получим новое решение $\Psi$ с $e(\Psi)=2\left(j_{0}-1\right)+1$ и $r(\Psi) \leqslant 2$ (см. [5], п. Б утверждения 13). Если $r(\Psi)=2$, то по индуктивному предположению $\Psi$ принадлежит некоторой сфере $S_{s t}^{2}$ с $s+t+1=e(\Phi)-2$. Более того, в этом случае переход от $\Psi$ к $\Phi$ осуществляется добавлением канонического положительного унитона, так что $\Phi$ принадлежит $S_{s+1, t+1}^{2}$ согласно п. Б. Если же $r(\Psi)=1$, то в силу замечания 2 можно (после некоммутативного сдвига) считать, что $\Psi=I-2 P_{0}$ c im $P_{0}=\left\langle e_{0}, \ldots, e_{m}\right\rangle$, где $m=2 j_{0}-1$. Тогда $\Phi$ принадлежит $S_{0, m+1}^{2}$ по доказанной выше лемме. Этим доказан шаг индукции. Доказательство для четных значений $e(\Phi)$ полностью аналогично. Утверждение доказано.

Утверждение 5 дает следующее описание пространств $\mathcal{M}(e, 2, u)$.

УтвеРЖДЕНИЕ 6. Для всех $е \geqslant 3$ имеем $\mathcal{M}(e, 2,2)=A_{e} \times \mathbb{C}_{\lambda}^{1}$, где $\mathbb{C}_{\lambda}^{1}$ nараметризует некоммутативные сдвиги, а $A_{e}$ получается из обгединения сфер $S_{s t}^{2}$ по всем иелым $s, t c 0 \leqslant s \leqslant t-2$ u $s+t+1=е$ удалением точки $(k, l)=(-1,0)$ сберы $S_{0, e-1}^{2}$. множество $\mathcal{M}(e, 2, u)$ пусто при $u \geqslant 3$.

ЗАмечание 6 . Опишем множества $A_{e}$ с геометрической точки зрения. При этом заменим букву $е$ на $m$ во избежание конфликта обозначений. Для любого $m \geqslant 3$ мно- 
жество $A_{m}$ является объединением $g:=[(m-1) / 2]$ двумерных сфер $S_{j}:=S_{j-1, m-j}^{2}$, $j=1,2, \ldots, g$, из которого удалена одна точка сферы $S_{1}$. Каждая сфера $S_{j}$ состоит из неграссмановых решений, за исключением двух своих “полюсов" $p_{j}, p_{j+1} \in S_{j}$, где точка $p_{j}$ представляет собой диагональное грассманово решение с образом $\left\langle e_{j-1}, \ldots, e_{m-j}\right\rangle$. Как и в разделе 3 , можно рассматривать $S_{j}$ как “комплексную пленку", интерполирующую между грассмановыми решениями $p_{j}, p_{j+1}$. Точка $p_{1} \in$ $S_{1}$ не принадлежит $A_{m}$, поскольку она представляет собой BPS-решение $(u=1)$. Имеем $S_{j} \cap S_{j+1}=\left\{p_{j+1}\right\}$ при каждом $j=1,2, \ldots, g$, а все остальные сферы $S_{j}$ попарно не пересекаются. Поэтому множество $A_{m}$ есть цепочка из $g$ двумерных сळер, последовательно соединяющих точки $p_{1}, \ldots, p_{g}$ и не имеющих других точек пересечения. Можно было бы еще представить $A_{m}$ как объединение $g$ непересекающихся экземпляров $S_{j} \backslash\left\{p_{j}\right\}$ плоскости $\mathbb{C}^{1}$, но это несколько затемняет структуру особенностей алгебраического многообразия $A_{m}$ в точках $p_{j}$.

\section{6. РЕШЕНИЯ С $e=4$}

Согласно замечанию 2 для любого не-BPS-решения $\Phi \in \mathcal{M}$ энергии $e(\Phi)=4$ имеем либо $r(\Phi)=3$, либо $r(\Phi)=2$. Из п. Б и В утверждения 2 видно, что случай $r(\Phi)=3$ имеет место тогда и только тогда, когда $\Phi$ принадлежит (с точностью до некоммутативного сдвига и эквивалентности) сфере (3.1), отвечающей $G=\left\langle e_{1}, R_{\alpha}-e_{0}\right\rangle$ и $B=\left\langle e_{0}, e_{1}, e_{2}, R_{\alpha}\right\rangle$ для некоторого $\alpha \in \mathbb{C} \backslash\{0\}$. Поэтому $\mathcal{M}(4,3,2) \cong \mathbb{C}_{\lambda}^{1} \times\left(\mathbb{C}_{\alpha}^{1} \backslash\{0\}\right) \times \mathbb{C}_{c}^{1}$, где $\lambda$ параметризует некоммутативные сдвиги, а $c$ - все не-BPS-решения вида (3.1) при каждом фиксированном $\alpha \in \mathbb{C} \backslash\{0\}$. Из утверждения 6 видно, что случай $r(\Phi)=2$ имеет место тогда и только тогда, когда $\Phi$ принадлежит (с точностью до некоммутативного сдвига и эквивалентности) сфере $S_{03}^{2}$, задаваемой формулой (5.1) для $s=0, t=3$, причем из этой сферы удалена одна точка. Таким образом, $\mathcal{M}(4,2,2) \cong \mathbb{C}_{\lambda}^{1} \times \mathbb{C}_{c}^{1}$, где $c \in \mathbb{C}$ параметризует все не-BPS-решения вида (5.1) с $s=0, t=3$ (связь между $c$ и $(k, l)$ задается формулами (4.1)). Этим доказан п. А следующего утверждения.

УТВЕРЖДЕНИЕ 7. А. С точностъю до некоммутативного сдвига и эквивалентности каждое не-BPS-решение $\Phi \in \mathcal{M}$ с $е(\Phi)=4$ задается либо формулой $\Phi=$ $\left(I-2 P^{0}\right)(I-2 Q(c))$, где $\operatorname{im} P^{0}:=\left\langle e_{0}\right\rangle u \operatorname{im} Q(c):=\left\langle e_{1}, e_{2}, e_{0}+c e_{3}\right\rangle$ для всех $c \in \mathbb{C}$ (это в точности формула (5.1) при $s=0, t=3, k \neq-1$ ), либо формулой $\Phi=$ $\left(I-2 P^{0}\right)(I-2 P(c, \alpha))$, дде $\operatorname{im} P(c, \alpha):=\left\langle e_{1}, e_{0}+c e_{2}, R_{\alpha}-e_{0}\right\rangle$ для всех $c \in \mathbb{C} u \alpha \in$ $\mathbb{C} \backslash\{0\}$ (это частный случай формуль (3.1)). Таким образом, имеем $\mathcal{M}(4,2,2) \cong \mathbb{C}^{2}$ u $\mathcal{M}(4,3,2) \cong \mathbb{C}^{3} \backslash \mathbb{C}^{2}$.

Б. Множество $\mathcal{M}(4,3,2) \cup \mathcal{M}(4,2,2)$ всех не-BPS-решений энергии $е(\Phi)=4$ является вещественно-аналитическим подмногообразием в $U(H)$, изоморфным $\mathbb{C}^{3}$. Его замыкание в $U(H)$ есть вещественно-аналитическое подмногообразие, изоморбное $\mathbb{C} P^{1} \times \mathbb{C}^{2}$. Переход $\kappa$ этому замыканию осуществляется добавлением некотоpых BPS-решений энергии $е(\Phi)=4$ и канонического ранга $r(\Phi) \leqslant 2$. 
ДокАзАТЕльство. Осталось доказать п. Б. Пользуясь определением (2.1), представим все элементы множества $\mathcal{M}(4,2,2)$ как пределы элементов $\mathcal{M}(4,3,2)$ по формуле $^{4)}$

$$
Q(c)=\lim _{\alpha \rightarrow 0} P\left(-\frac{\sqrt{3} c}{\alpha}, \alpha\right) .
$$

Это позволяет нам вещественно-аналитически и взаимно однозначно параметризовать замыкание множества $\mathcal{M}(4,3,2) \cup \mathcal{M}(4,2,2)$ в $U(H)$ посредством многообразия $\mathbb{C} P^{1} \times \mathbb{C}^{2}$. Действительно, покроем $\mathbb{C} P^{1} \times \mathbb{C}^{2}$ двумя координатными картами $\left(c^{\prime}, \alpha, \lambda\right) \in \mathbb{C}^{3}$ и $\left(c^{\prime \prime}, \alpha, \lambda\right) \in \mathbb{C}^{3}$ с функцией перехода $c^{\prime \prime}=1 / c^{\prime}$. Вложим первую карту в $U(H)$ по формуле $\Phi\left(c^{\prime}, \alpha, \lambda\right):=U_{\lambda}\left(I-2 P^{0}\right)\left(I-2 P_{1}\left(c^{\prime}, \alpha\right)\right) U_{\lambda}^{*}$, где $U_{\lambda}$ есть некоммутативный сдвиг (см. раздел 3$)$, а ортопроектор $P_{1}\left(c^{\prime}, \alpha\right)$ задан формулой $\operatorname{im} P_{1}\left(c^{\prime}, \alpha\right):=\left\langle e_{1}, e_{0}+c^{\prime} e_{3}+\sqrt{3} c^{\prime} \alpha M(\alpha), e_{2}+\frac{\alpha e_{3}}{\sqrt{3}}+\alpha^{2} M(\alpha)\right\rangle=\operatorname{im} P\left(-\frac{\sqrt{3} c^{\prime}}{\alpha}, \alpha\right)$, причем

$$
M(\alpha):=\frac{\sqrt{2}}{\alpha^{4}}\left(R_{\alpha}-\sum_{j=0}^{3} \frac{\alpha^{j} e_{j}}{\sqrt{j !}}\right)
$$

есть голоморфная $H$-значная функция от $\alpha \in \mathbb{C}$ с $M(0)=e_{4} / \sqrt{12}$. Вторая карта аналогично вкладывается в $U(H)$ по формуле

$$
\Psi\left(c^{\prime \prime}, \alpha, \lambda\right):=\Phi\left(\frac{1}{c^{\prime \prime}}, \alpha, \lambda\right)=U_{\lambda}\left(I-2 P^{0}\right)\left(I-2 P_{2}\left(c^{\prime \prime}, \alpha\right)\right) U_{\lambda}^{*},
$$

где

$$
\operatorname{im} P_{2}\left(c^{\prime \prime}, \alpha\right):=\left\langle e_{1}, c^{\prime \prime} e_{0}+e_{3}+\sqrt{3} \alpha M(\alpha), e_{2}+\frac{\alpha e_{3}}{\sqrt{3}}+\alpha^{2} M(\alpha)\right\rangle .
$$

Векторы, порождающие подпространства im $P_{1}\left(c^{\prime}, \alpha\right)$ и im $P_{2}\left(c^{\prime \prime}, \alpha\right)$, всегда линейно независимы, поскольку уже их проекции на $\left\langle e_{0}, e_{1}, e_{2}\right\rangle$ таковы. Теперь ясно, что замыкание множества $\mathcal{M}(4,3,2) \cup \mathcal{M}(4,2,2)$ в $U(H)$ получается добавлением к этому множеству следующих семейств BPS-решений: $\mathcal{M}(4,1,1) \cong \mathbb{C}^{1}$ и $\mathcal{M}_{1} \cong \mathbb{C}^{2} \backslash \mathbb{C}^{1}$ (в обозначениях замечания 3). Множество $\mathcal{M}_{1}$ состоит из всех BPS-решений с кратностями 3,1 и является одной из двух связных компонент пространства $\mathcal{M}(4,2,1)$. Утверждение доказано.

\section{7. РЕШЕНИЯ С $e=5$}

Множества всех не-BPS-решений $\Phi \in \mathcal{M}_{0}$ энергии $e(\Phi)=5$ и канонического ранга $r=4$ или $r=2$ уже известны: мы имеем $\mathcal{M}(5,4,2) \cong D^{3} \times \mathbb{C}^{1}$ для некоторого открытого подмножества $D^{3} \subset \mathbb{C}^{3}$ (см. замечание 5) и $\mathcal{M}(5,2,2) \cong \mathbb{C}^{1} \times\left(S_{13}^{2} \cup S_{04}^{2} \backslash\{\mathrm{pt}\}\right)$ (см. замечание 6). Кроме того, п. Б утверждения 2 описывает две связные компоненты множества $\mathcal{M}(5,3,2)$. Обозначим их через $\mathcal{N}_{j} \cong \mathcal{B}_{j} \times \mathbb{C}_{c}^{1}, j=1,2$. Здесь $\mathcal{B}_{1}, \mathcal{B}_{2} \cong \mathbb{C}^{2} \backslash \mathbb{C}^{1}$ - две связные компоненты множества $\mathcal{N}(5,2,1)$ (см. п. В утверждения 1$)$, а каждое $\mathcal{N}_{j}$ состоит из всех не-BPS-решений вида (3.1), для которых $B \in \mathcal{B}_{j}$. Следующее утверждение завершает описание полной картины.

\footnotetext{
${ }^{4)}$ Если просто фиксировать $c \in \mathbb{C}$ и устремить $\alpha \rightarrow 0$, то предел проекторов $P(c, \alpha)$ будет равен $Q(0)$ независимо от значения $c$.
} 
УтВеРЖДЕНИЕ 8. А. Каждое не-ВPS-решение $\Phi \in \mathcal{M}_{0}$ с e $e(\Phi)=5$ u $r(\Phi)=3$ либо принадлежит $\mathcal{N}_{1} \cup \mathcal{N}_{2}$, либо имеет вид

$$
\Phi=U_{\lambda}\left(I-2 P_{0}\right)\left(I-2 P\left(c_{1}, c_{2}\right)\right) U_{\lambda}^{*}
$$

для некоторьх $\lambda \in \mathbb{C}, c_{1} \in \mathbb{C} \backslash\{0\}, c_{2} \in \mathbb{C}$, где $U_{\lambda}$ есть некоммутативный сдвиг, a $P_{0}, P\left(c_{1}, c_{2}\right)$ - ортопроекторы, заданные формулами

$$
\operatorname{im} P_{0}=\left\langle e_{0}, e_{1}\right\rangle, \quad \operatorname{im} P\left(c_{1}, c_{2}\right)=\left\langle e_{2}, e_{0}+c_{1} e_{3}, e_{1}+c_{2} e_{3}+\frac{c_{1} e_{4}}{2}\right\rangle .
$$

Обозначим множество всех таких операторов (7.1) через $\mathcal{N}_{3}$. Тогда имеем $\mathcal{M}(5,3,2)=\mathcal{N}_{1} \cup \mathcal{N}_{2} \cup \mathcal{N}_{3}$. Множества $\mathcal{N}_{1}, \mathcal{N}_{2}, \mathcal{N}_{3}$ являются непересекаюшимися незамкнутыми вещественно-аналитическими подмногообразиями в $U(H)$, изоморфными $\mathbb{C}^{3} \backslash \mathbb{C}^{2}$.

Б. Замыкания множеств $\mathcal{N}_{j}$ при $j=1,2$ получаются добавлением $\kappa$ ним множеств $\mathcal{B}_{j} \cup\left(\mathbb{C}^{1} \times S_{04}^{2}\right) \subset \mathcal{M}(5,2,1) \cup \mathcal{M}(5,1,1) \cup \mathcal{M}(5,2,2)$ и изоморфны $\mathbb{C P}^{1} \times \mathbb{C}^{2}$. Замыкание множества $\mathcal{N}_{3}$ получается добавлением $\kappa$ нему множества $\mathbb{C}^{1} \times\left(S_{04}^{2} \cup\right.$ $\left.S_{13}^{2}\right)=\mathcal{M}(5,1,1) \cup \mathcal{M}(5,2,2)$ и изоморфно прямому произведению $\mathbb{C}^{1}$ на некоторую компактную комплексную поверхность с одной особой точкой типа конуса.

ДоказАтельство. А. Добавляя к $\Phi$ канонический отрицательный $\Phi$-унитон, получим новое решение $\Psi$ с $e(\Psi)=2$. Согласно замечанию 2 можно считать, что $\Psi=$ $I-2 P_{D}$, где $P_{D}$ есть ортопроектор на некоторое BPS-подпространство $D \subset H$ размерности 2. Из п. А утверждения 13 работы [5] вытекает, что $\Phi=\left(I-2 P_{D}\right)(I-2 P)$ для некоторого ортопроектора $P$ с $\operatorname{dim} \operatorname{im} P=3$, удовлетворяющего условиям (1.3) с заменой $\Phi$ на $\Psi=I-2 P_{D}$. Согласно формуле (3.13) из [5] эти условия на пространство $V:=\operatorname{im} P$ имеют вид

$$
E \ominus D \subset V, \quad\left(P_{D} a P_{D}+P_{D}^{\perp} a P_{D}^{\perp}\right) V \subset V
$$

где BPS-решение $E \supset D$ размерности $2+r(\Psi)$ определяется как образ BPS-решения $D$ при добавлении канонического положительного $\Psi$-унитона (тогда im $\Psi_{+}=E \ominus D$ в силу замечания 1$)$. Применяя $P_{D}$ к обеим частям второго условия (7.3) и пользуясь инвариантностью $D$ относительно $a$, получаем, что $a P_{D} V \subset P_{D} V$. Таким образом, векторное пространство $P_{D} V \subset D$ является $B P S$-подпространством в $H$. Его размерность может быть равна 0,1 или 2. Покажем, что первый случай невозможен, второй дает $\mathcal{N}_{1} \cup \mathcal{N}_{2}$, а третий дает $\mathcal{N}_{3}$.

Случай $1 . \operatorname{dim} P_{D} V=0$. Тогда $V \perp D$ и исходное решение $\Phi=\left(I-2 P_{D}\right)(I-2 P)$ равно $I-2 P_{D \oplus V}$. При этом второе условие (7.3) принимает вид $a V \subset D \oplus V$, откуда $a(D \oplus V) \subset D \oplus V$, и $\Phi$ является BPS-решением вопреки предположению. Следовательно, случай 1 невозможен.

Случай 2. $\operatorname{dim} P_{D} V=1$. Тогда векторное подпространство $V^{\prime}:=\left\{x \in V \mid P_{D} x=\right.$ $0\}=V \cap D^{\perp}$ имеет коразмерность 1 в $V$, т.е. $\operatorname{dim} V^{\prime}=2$. После некоммутативного сдвига можно считать, что одномерное BPS-подпространство $P_{D} V$ натянуто на вектор $e_{0} \in D$. Значит, вектор нормали $u$ к $V^{\prime}$ в $V$ можно записать в виде 
$u=e_{0}+\xi$, где $\xi \in D^{\perp}$. Заметим, что $E \ominus D \subset V^{\prime}$ в силу первого условия (7.3) и положим $V_{1}:=V^{\prime} \ominus(E \ominus D)$. Тогда второе условие (7.3) принимает вид $P_{D}^{\perp} a V_{1} \subset V^{\prime}$, $P \underset{D}{\perp} a \xi \subset V^{\prime}$ или, эквивалентно,

$$
a V_{1} \subset M \quad \text { и } \quad a \xi \in M, \quad \text { где } \quad M:=D \oplus V^{\prime}=E \oplus V_{1} .
$$

Отсюда $\operatorname{dim} M=4$, и $M$ является BPS-подпространством. Поскольку $D \ni e_{0}$, имеем $E \supset\left\langle e_{0}, e_{1}\right\rangle$. Поэтому $G:=M \ominus\left\langle e_{0}\right\rangle$ является грассмановым решением энергии $e(\Phi)=5$ и дефекта 2 (см. начало раздела 3$)$.

Если $\xi=0$, то исходное решение $\Phi=\left(I-2 P_{D}\right)(I-2 P)$ совпадает с $I-2 P_{G}$. Поскольку $r(\Phi)=3$, из п. Б утверждения 2 вытекает, что $\Phi \in \mathcal{N}_{1} \cup \mathcal{N}_{2}$. Поэтому будем считать, что $\xi \neq 0$. Тогда $B:=M \oplus\langle\xi\rangle$ является BPS-подпространством размерности 5 (это вытекает из (7.4) и того, что $\xi \perp M$ согласно определениям $\xi$ и $M)$. Пусть $k \geqslant 2$ есть наибольшее целое число такое, что $e_{0}, e_{1}, \ldots, e_{k-1} \in M$. Тогда $\xi=c P_{M}^{\perp} e_{k}$ для некоторого $c \in \mathbb{C} \backslash\{0\}$ (чтобы убедиться в этом, заметим, что все прообразы всех базисных векторов (2.4) пространства $M$ относительно $a$ лежат опять в $M$, за исключением $a^{-1}\left(e_{k-1}\right)=e_{k} / \sqrt{k}+$ const $\left.\cdot e_{0} \cdot\right)$ Отсюда имеем $B=M \oplus\left\langle e_{k}\right\rangle$, что согласуется с обозначениями раздела 3 . Полагая $N:=P_{M}^{\perp} e_{k}$, мы видим, что $\xi=c N, V=V^{\prime} \oplus\left\langle e_{0}+c N\right\rangle$ и $B \ominus G=\left\langle e_{0}, N\right\rangle$. Теперь прямая проверка, использующая (7.4) и очень похожая на доказательство утверждения 3 , показывает, что $\Phi x=x$ для $x \in B^{\perp}, \Phi x=-x$ для $x \in G$, а сужение $\Phi$ на $B \ominus G$ задается матрицей (3.6) в ортонормированном базисе $\left\langle e_{0}, N /|N|\right\rangle$ пространства $B \ominus G$. Таким образом, решение $\Phi$ принадлежит сфере (3.1), порожденной грассмановым решением $G$. Поскольку $r(\Phi)=3$, из п. Б утверждения 2 получаем, что $\Phi \in \mathcal{N}_{1} \cup \mathcal{N}_{2}$.

Случай 3. $\operatorname{dim} P_{D} V=2$. Тогда подпространство $V^{\prime}:=V \cap D^{\perp}$ имеет коразмерность 2 в $V$, т.е. $\operatorname{dim} V^{\prime}=1$. Из включения $V^{\prime} \supset E \ominus D$ вытекает, что число $\operatorname{dim}(E \ominus D)=r(\Psi)$ тоже равно единице. Поэтому после некоммутативного сдвига можно считать, что $D=\left\langle e_{0}, e_{1}\right\rangle$ и $E \ominus D=\left\langle e_{2}\right\rangle=V^{\prime}$. Так как $P_{D} V=V$, то оставшиеся базисные векторы пространства $V$, ортогональные к $V^{\prime}$, можно записать в виде $e_{0}+\xi_{0}$ и $e_{1}+\xi_{1}$, где $\xi_{0}, \xi_{1} \in\left\langle e_{0}, e_{1}, e_{2}\right\rangle^{\perp}$. Второе условие (7.3) принимает вид

$$
P_{D}^{\perp} a \xi_{0} \in V, \quad e_{0}+P_{D}^{\perp} a \xi_{1} \in V .
$$

Отсюда получаем $a \xi_{0} \in V^{\prime}$. Поскольку $V^{\prime}=\left\langle e_{2}\right\rangle$ и $\xi_{0} \in\left\langle e_{0}, e_{1}, e_{2}\right\rangle^{\perp}$, мы видим, что $\xi_{0}=c_{1} e_{3}$ для некоторого $c_{1} \in \mathbb{C}$. Второе включение $(7.5)$ можно записать в виде $e_{0}+P_{D}^{\perp} a \xi_{1}=A e_{2}+B\left(e_{0}+\xi_{0}\right)+C\left(e_{1}+\xi_{1}\right)$ для некоторых $A, B, C \in \mathbb{C}$. Сравнивая проекции обеих частей этого равенства на $\left\langle e_{0}\right\rangle$, а затем на $\left\langle e_{0}, e_{1}\right\rangle$, мы находим $B=1$ и $C=0$. Следовательно, $\xi_{1}=c_{1} e_{4} / 2+c_{2} e_{3}$ для некоторого $c_{2} \in \mathbb{C}$. Тем самым $V$ совпадает с пространством $\operatorname{im} P\left(c_{1}, c_{2}\right)$ из (7.2), а оператор $\Phi$ задается формулой (7.1) с $\lambda=0$. Случай $c_{1}=0$ следует исключить, поскольку операторы $(7.1)$ с $c_{1}=0$ и $\lambda=0$ имеют канонический ранг $r(\Phi)=2$ (они образуют сферу $S_{13}^{2}$ в обозначениях раздела 5). Все остальные решения (7.1) с $\lambda=0$ имеют канонический ранг $r(\Phi)=3$. Действительно, они не могут принадлежать $\mathcal{M}(5,4,2)$, поскольку действуют внутри BPS-подпространства с только одним корнем, и не могут принадлежать оставшейся сфере $S_{04}^{2} \subset \mathcal{M}(5,2,2)$, поскольку не переводят $e_{1}$ в $-e_{1}$. 
Б. Утверждения о замыканиях множеств $\mathcal{N}_{1}$ и $\mathcal{N}_{2}$ следуют из результатов раздела 3. Чтобы найти замыкание $\mathcal{N}_{3}$, рассмотрим множество $\mathcal{N}_{3}^{2}$ всех операторов (7.1) с $\lambda=0$. Его замыкание может дополнительно содержать только элементы сфер $S_{04}^{2}$ и $S_{13}^{2}$. И обе эти сферы действительно содержатся в указанном замыкании: вводя обозначение $\Phi_{0}=I-2 P_{G}$ для $G=\left\langle e_{1}, e_{2}, e_{3}\right\rangle$, мы видим, что $S_{13}^{2} \backslash\left\{\Phi_{0}\right\}$ есть координатная ось $\left\{c_{1}=0\right\}$ в обозначениях (7.2), а $S_{04}^{2} \backslash\left\{\Phi_{0}\right\}-$ координатная ось $\left\{c_{1}^{\prime}=0\right\}$ пространства $\mathbb{C}^{2}$ с координатами $c_{1}^{\prime}:=c_{1}^{-1}, c_{2}^{\prime}:=2 c_{1}^{-1}\left(1-c_{2} c_{1}^{-1}\right)$, где

$$
\operatorname{im} P\left(c_{1}, c_{2}\right)=\left\langle e_{2}, e_{3}+c_{1}^{\prime} e_{0}, e_{4}+c_{2}^{\prime} e_{0}+2 c_{1}^{\prime} e_{1}\right\rangle .
$$

Далее будем обозначать замыкание $\mathcal{N}_{3}^{2}$ через $\mathcal{R}^{2}$. Из (7.6) и (7.2) видно, что все точки комплексной поверхности $\mathcal{R}^{2}=\mathcal{N}_{3}^{2} \cup S_{13}^{2} \cup S_{04}^{2}$ гладкие, кроме, может быть, $\Phi_{0}$. Чтобы убедиться в том, что точка $\Phi_{0}$ является особой, положим $\gamma_{1}:=c_{1}$ и $\gamma_{2}:=c_{2}^{-1}$. Тогда

$$
\operatorname{im} P\left(c_{1}, c_{2}\right)=\left\langle e_{2}, e_{0}+A e_{1}+B e_{4}, e_{3}+C e_{1}+D e_{4}\right\rangle,
$$

где $A=-\gamma_{1} \gamma_{2}, B=-\gamma_{1}^{2} \gamma_{2} / 2, C=\gamma_{2}, D=\gamma_{1} \gamma_{2} / 2$. Поэтому поверхность $\mathcal{R}^{2}$ задается уравнениями $A+2 D=0, A D=B C$ в пространстве $\mathbb{C}^{4}$ с координатами $A, B, C, D$. Начало координат $\Phi_{0}$ пространства $\mathbb{C}^{4}$ является особой точкой типа конуса на $\mathcal{R}^{2}$ (см. замечание 8 ниже). Этим утверждение п. Б доказано, поскольку замыкание множества $\mathcal{N}_{3}$ равно $\mathcal{R}^{2} \times \mathbb{C}_{\lambda}^{1}$.

ЗАмечАниЕ 7. Обобщая описание случая 2 из доказательства п. А утверждения 8, можно показать, что все решения $\Phi \in \mathcal{M}_{0}$ с $r(\Phi)=e(\Phi)-2$ и $\operatorname{dim} P_{D} V=1$ принадлежат некоторым сферам (3.1) с точностью до некоммутативного сдвига. Описание случая 3 также допускает обобщение, в результате которого мы получаем двумерную "комплексную пленку" решений с $r=e-2$, интерполирующую между BPS-решением $B=M_{1} \oplus\left\langle e_{4}\right\rangle=M_{2} \oplus\left\langle e_{3}, e_{4}\right\rangle$ энергии $е$ и двумя грассмановыми решениями $G_{1}=M_{1} \ominus\left\langle e_{0}\right\rangle, G_{2}=M_{2} \ominus\left\langle e_{0}, e_{1}\right\rangle$ энергии $е$ и дефекта 2,4 соответственно. Эта конструкция представляет собой следующий по сложности шаг по сравнению с конструкциями раздела 3. Если энергия $е$ достаточно велика, то можно пойти дальше и получить “комплексные пленки" более высокой размерности, интерполирующие между элементами большего множества грассмановых решений.

ЗАмечАниЕ 8. Комплексная поверхность $\mathcal{R}^{2}$ биголоморфна проективной компактификации стандартного конуса $\left\{(x, y, z) \in \mathbb{C}^{3} \mid x^{2}+y^{2}=z^{2}\right\}$, вершина которого соответствует решению $\Phi_{0}=I-2 P_{G}$. В этом легко убедиться, полагая $A=-\sqrt{2} x$, $B=y-z, C=y+z, D=x / \sqrt{2}$. Поскольку замыкания всех $\mathcal{N}_{j}, j=1,2,3$, содержат $\mathbb{C}_{\lambda}^{1} \times S_{04}^{2}$, можно представлять себе замыкание множества $\mathcal{M}(5,3,2)$ в виде "книги" с тремя страницами $\overline{\mathcal{N}}_{1}, \overline{\mathcal{N}}_{2}, \overline{\mathcal{N}}_{3}$ (комплексной размерности 3 ) и "переплетом" $\mathbb{C}^{1} \times \mathbb{C} P^{1}$. Заметим еще, что формулы (7.2), (7.6) и (7.7) представляют собой не что иное, как координатные представления поверхности $\mathcal{R}^{2}$ в трех различных стандартных картах грассманова многообразия $\mathrm{Gr}_{2}\left(\mathbb{C}^{4}\right)$, где $\mathbb{C}^{4}=\left\langle e_{0}, e_{1}, e_{3}, e_{4}\right\rangle$.

ЗАмЕчАниЕ 9. Полученные результаты подтверждают гипотезу о реализуемых нулевых модах гессиана функционала энергии (см. [5], замечание 10) для случая $e \leqslant 5$. Действительно, обозначим через $\Phi_{0}$ диагональное BPS-решение энергии $e$. 
Тогда $r\left(\Phi_{0}\right)=1$. Если $e \in\{3,4\}$, то единственное комплексное касательное направление в точке $\Phi_{0}$ к пространству неграссмановых решений есть касательное пространство к $S_{0, e-1}^{2} \subset \mathcal{M}(e, 2,2)$. Если же $e=5$, то реализуемые нулевые моды гессиана функционала энергии в точке $\Phi_{0}$ образуют комплексное двумерное касательное пространство к комплексной поверхности $\mathcal{R}^{2}$ в этой точке. Для любых значений $e$ формулы (4.5), (4.6) работы [5] задают $g:=[(e-1) / 2]$ линейно независимых комплексных нулевых мод, линейная оболочка которых совпадает с касательным пространством в точке $\Phi_{0}$ к $g$-мерной "комплексной пленке", упомянутой в замечании 7. Задача заключается в доказательстве отсутствия других линейно независимых касательных направлений в точке $\Phi_{0}$ к пространству неграссмановых решений энергии $e$. Эта задача остается нерешенной для $e \geqslant 6$.

\section{8. ЗАКЛЮЧЕНИЕ}

Обозначим через $\mathcal{M}(e) \subset U(H)$ множество всех решений $\Phi \in \mathcal{M}_{0}$ с $e(\Phi)=e$. Мы показали, что множества $\mathcal{M}(1), \mathcal{M}(2), \ldots, \mathcal{M}(5)$ равны соответственно

$$
\mathbb{C}^{1}, \quad \mathbb{C}^{2}, \quad \mathbb{C}^{3} \cup A_{1}^{2}, \quad \mathbb{C}^{4} \cup A_{1}^{3}, \quad \mathbb{C}^{5} \cup A_{1}^{4} \cup A_{2}^{3},
$$

где $\mathbb{C}^{e}-$ множество всех BPS-решений энергии $e$ (см. раздел 2), а каждое $A_{s}^{k}-$ некоторое неприводимое алгебраическое многообразие размерности $k$ над $\mathbb{C}$. Здесь и далее имеется в виду, что все эти многообразия вещественно-аналитически вложены в $U(H)$. Если $e \in\{3,4\}$, то многообразия $A_{1}^{e-1} \cong \mathbb{C} P^{1} \times \mathbb{C}^{e-2}$ и их пересечения $A_{1}^{e-1} \cap \mathbb{C}^{e} \cong \mathbb{C}^{e-2}$ с BPS-компонентой являются гладкими (см. разделы 4,6$)$. Однако алгебраические многообразия $A_{1}^{4} \cong \mathbb{C}^{1} \times M^{3}$ и $A_{2}^{3} \cong \mathcal{R}^{2} \times \mathbb{C}^{1}$ уже не гладкие (см. замечания 5 и 8). При этом $\mathbb{C}^{5} \cap A_{1}^{4} \cong M^{3}, \mathbb{C}^{5} \cap A_{2}^{3} \cong \mathbb{C}^{1}$ и $A_{1}^{4} \cap A_{2}^{3} \cong \mathbb{C}^{1} \times \mathbb{C P}^{1}$. $\mathrm{B}$ частности, множества $\mathcal{M}(e)$ линейно связны при $e \leqslant 5$.

Мы имеем также полное описание множеств $\mathcal{M}(e, r, u)($ см. (1.5)) для всех случаев, когда $u=1$ или $r \in\{1,2, e-1, e\}$ (см. п. В утверждения 1 и замечания 2, $5,6): \mathcal{M}(e, r, 1)$ есть конечное дизъюнктное объединение открытых связных подмножеств $\mathbb{C}^{r}$ при $1 \leqslant r \leqslant e, \mathcal{M}(e, e-1,2)$ есть открытое связное подмножество в $\mathbb{C}^{e-1}$, $\mathcal{M}(e, 2,2)$ есть прямое произведение $\mathbb{C}^{1}$ на цепочку из $[(e-1) / 2]$ экземпляров $\mathbb{C P}^{1}$, из которой удалена одна точка, а все остальные множества $\mathcal{M}(e, r, u)$ в указанном диапазоне значений $e, r, u$ являются пустыми. Вне этого диапазона известно только то, что $\mathcal{M}(5,3,2)$ есть объединение трех непересекающихся экземпляров $\mathbb{C}^{3} \backslash \mathbb{C}^{2}$ и что все множества $\mathcal{M}(e, e-2, u)$ пусты при $u \geqslant 3$.

Эти результаты позволяют высказать следующие гипотезы, которые не только указывают возможные направления для дальнейших исследований, но и суммируют основные моменты полученного в настоящей работе “экспериментального материала".

1. Имеем $\mathcal{M}(e)=\mathbb{C}^{e} \cup A_{1}^{e-1} \cup \cdots \cup A_{g}^{e-g}$, где $g=[(e-1) / 2], \mathbb{C}^{e}-$ множество BPS-решений, а каждое $A_{s}^{e-s}$ есть неприводимое алгебраическое многообразие размерности $e-s$ над $\mathbb{C}$, содержащее множество $\mathcal{M}(e, 1,1) \subset \mathbb{C}^{e}$. В частности, пространство $\mathcal{M}(e)$ всегда связно.

2. Множество $\mathcal{M}(e, r, u)$ либо пусто, либо $r$-мерно над $\mathbb{C}$. 
3. Любое решение $\Phi \in \mathcal{M}_{0}$ подчинено некоторому BPS-решению $\Phi_{0}=I-2 P_{0}$ той же энергии, т.е. имеем $\operatorname{dim} \operatorname{im} P_{0}=e(\Phi)$ и $\Phi x=x$ для всех $x \in\left(\operatorname{im} P_{0}\right)^{\perp}$.

4. Для всякого BPS-решения $\Phi_{0}$ множество всех подчиненных $\Phi_{0}$ решений $\Phi \in$ $\mathcal{M}(e)$ имеет размерность $\sum_{j=1}^{r}\left[\left(k_{j}-1\right) / 2\right]$ над $\mathbb{C}$ (где $k_{1}, \ldots, k_{r}$ суть кратности $\Phi_{0}$ в смысле замечания 1$)$, содержит $\Phi_{0}$ в качестве гладкой точки и является трансверсальным к множеству всех BPS-решений в этой точке.

5. Имеем $r(\Phi) \geqslant u(\Phi)$ для всех решений $\Phi$, причем для диагональных $\Phi$ (см. [5], п. 4.1) имеет место равенство. Множество $\mathcal{M}(e, r, u)$ непусто тогда и только тогда, когда $e \geqslant r \geqslant u$ и $2 e \geqslant u(u+1)$.

Благодарности. Автор благодарен профессору О. Лехтенфельду за интерес к работе и многочисленные полезные обсуждения. Работа выполнена в рамках совместного проекта RFFI-DFG 436 RUS 113/669. Она также поддержана грантами РФФИ (№ 05-01-00981, 05-01-00498) и Президентской программой поддержки ведущих научных школ (грант НШ-9429.2006.1).

\section{Список литературы}

[1] W. J. Zakrzewski, Low Dimensional Sigma Models, Adam Hilger, Bristol, 1989.

[2] Й. Давидов, А. Г. Сергеев, УМН, 48:3 (1993), 3-96.

[3] O. Lechtenfeld, A. D. Popov, JHEP, 11 (2001), 40.

[4] A. V. Domrin, O. Lechtenfeld, S. Petersen, JHEP, 03 (2005), 045.

[5] А. В. Домрин, ТМФ, 154:2 (2008), 220-239.

[6] J. Murugan, R. Adams, JHEP, 12 (2002), 073; O. Foda, I. Jack, D. R. T. Jones, Phys. Lett. B, 547:1-2 (2002), 79-84.

[7] А. М. Переломов, Обобщенные когерентные состояния и их применения, Наука, М., 1987.

[8] R. Rochberg, N. Weaver, Proc. Amer. Math. Soc., 129:9 (2001), 2679-2687.

[9] А. В. Комлов, ТМФ, 153:3 (2007), 347-357. 